\title{
Integrin $\alpha 7$ correlates with worse clinical features and prognosis, and its knockdown inhibits cell proliferation and stemness in tongue squamous cell carcinoma
}

\author{
ZHIYONG LV, YE YANG and CHUNYAN YANG \\ Department of Oral and Maxillofacial Surgery, The Second Affiliated Hospital of Harbin Medical University, \\ Harbin, Heilongjiang 150001, P.R. China
}

Received January 24, 2019; Accepted September 9, 2019

DOI: 10.3892/ijo.2019.4927

\begin{abstract}
The present study aimed to evaluate the correlation of integrin $\alpha 7$ (ITGA7) with clinicopathological characteristics and overall survival (OS) in patients with tongue squamous cell carcinoma (TSCC), and to investigate the effect of ITGA7 knockdown on proliferation, apoptosis and stemness of TSCC cells in vitro. ITGA7 expression was measured in tumor tissues and paired adjacent normal tissues from 60 patients with TSCC using immunohistochemistry. ITGA7 expression in human TSCC cell lines and normal oral keratinocytes was measured using quantitative PCR and western blotting. Lentiviruses carrying short hairpin (sh) RNA targeting ITGA7 were used to knockdown its expression in CAL-27 and HSC-4 cells, and then proliferation, apoptosis and stemness were measured. In addition, CAL-27 and HSC-4 cancer stem cells (CSCs) were constructed and their ITGA7 expression was measured. The results demonstrated that ITGA7 was upregulated in the tumor tissues compared with the paired adjacent tissues, and its high expression was correlated with worse pathological grade, $\mathrm{N}$ stage, TNM stage and OS. In vitro, ITGA7 expression levels were demonstrated to be increased in the TSCC CAL-27, SCC-9, HSC-4 and SCC-25 cell lines compared to the normal HOK cell line. In CAL-27 and HSC-4 cells, ITGA7 knockdown inhibited cell proliferation, promoted apoptosis, increased CD24 expression, decreased CD44 and CD133
\end{abstract}

Correspondence to: Dr Chunyan Yang, Department of Oral and Maxillofacial Surgery, The Second Affiliated Hospital of Harbin Medical University, 246 Xuefu Road, Harbin, Heilongjiang 150001, P.R. China

E-mail: canjian9747320347@163.com

Abbreviations: ITGA7, integrin $\alpha 7$; OS, overall survival; TSCC, tongue squamous cell carcinoma; CSCs, cancer stem cells; OSCC, oral squamous cell carcinoma; TNM, tumor-node-metastasis; MOI, multiplicity of infection

Key words: tongue squamous cell carcinoma, cancer stem cells, overall survival, sphere formation expression, reduced drug resistance to cisplatin and attenuated sphere formation efficiency. Finally, ITGA7 expression levels were greatly elevated in CAL-27 and HSC-4 CSCs compared with parental CAL-27 and HSC-4 cells. In conclusion, ITGA7 knockdown inhibited tumor cell proliferation and stemness in TSCC cells. These findings indicated that ITGA7 might serve as a potential marker for CSCs and may correlate with worse clinical features and prognosis in TSCC.

\section{Introduction}

Tongue squamous cell carcinoma (TSCC), one of the most common type of oral squamous cell carcinoma (OSCC) that ranks as the sixth leading cause of cancer-associated mortality worldwide, presents with more aggressive characteristics compared with other forms of OSCC; 17,110 new cases and 2,510 mortalities due to TSCC have been reported in the United States in 2018 (1,2). At present, surgical resection is considered as the primary treatment option for patients with TSCC; however, the majority of patients diagnosed with TSCC are of an advanced stage, at which point surgery cannot be performed. For patients who have undergone surgery, the inevitable oral complications, including speech impediments and swallowing dysfunction notably affect quality of life $(3,4)$. Although various treatments, including radiotherapy, chemotherapy and targeted therapy have been applied to patients with TSCC, unsatisfactory outcomes have been reported, partly due to distant metastasis, tumor recurrence and drug resistance $(4,5)$. Therefore, it is important to identify additional reliable biomarkers to accurately monitor the progression and predict the prognosis of patients with TSCC.

Integrins, a large family of heterodimeric cell surface receptors that participate in cell-extracellular matrix and cell-cell interactions, are involved in a broad range of cellular processes, such as cell growth, cell mobility and cell signaling networks $(6,7)$. As one member of the integrin family of adhesion molecules, integrin $\alpha 7$ (ITGA7), located on chromosome 12 p13 and comprising $>27$ exons spanning a region of $\sim 22.5 \mathrm{~kb}$, has been reported to be a tumor suppressor in several carcinomas, including prostate cancer (8), breast cancer (9) and melanoma (10). By contrast, ITGA7 was determined to act as a tumor promoter in OSCC (11) and glioblastoma (12). 
In addition, ITGA7 has been reported as a functional marker of cancer stem cells (CSCs) and serves a critical role in the regulation of stem cell-like properties in a variety of cancer cells $(11,13)$. Several previous studies investigated the potential role of ITGA7 in the pathogenesis of different types of carcinomas; however, the specific function of ITGA7 in TSCC remains unknown.

Based on the aforementioned reports regarding the regulatory roles of ITGA7 in tumor progression and the properties of CSCs in carcinoma, we hypothesized that ITGA7 may act as a critical regulator in the pathogenesis of TSCC. The present study aimed to detect the expression of ITGA7 in tumor and paired adjacent tissues, and to evaluate its association with the clinicopathological characteristics and overall survival (OS) of patients with TSCC. Additionally, the effects of ITGA7 knockdown were investigated on the proliferation, apoptosis and stemness of TSCC cells.

\section{Materials and methods}

Patients. A total of 60 patients with TSCC who underwent surgical treatment in The Second Affiliated Hospital of Harbin Medical University between January 2014 and December 2016 were included in the present study. The inclusion criteria were: i) Patients with a clinically and histopathologically confirmed diagnosis of TSCC; ii) received surgical resection as initial treatment; iii) archived tumor tissue and paired adjacent tissues that were stored at the Pathological Department of the hospital; and iv) clinicopathological and follow-up data were complete and accessible. Patients were excluded if they underwent radiotherapy or chemotherapy prior to surgery. The present study was approved by the Institutional Review Board of The Second Affiliated Hospital of Harbin Medical University, and written informed consent was obtained from all patients or their guardians.

Data collection. The demographic data and clinicopathological characteristics of patients, including age, gender, pathological grade, T stage, $\mathrm{N}$ stage, and tumor-node-metastasis (TNM) stage, which was evaluated according to the criteria of the 7 th edition American Joint Committee on Cancer, were obtained from medical records. The survival data, which were used to calculate OS, were obtained from follow-up records (last follow-up date was 30/06/2018). The OS was defined as the duration from surgical treatment to patient mortality.

ITGA7 and CD133 expression in TSCC and paired adjacent tissues. Immunohistochemistry and reverse transcription-quantitative polymerase chain reaction (RT-qPCR) were performed to detect ITGA7 expression levels; immunohistochemistry was also conducted to detect CD133 expression levels in TSCC and paired adjacent tissues.

Immunohistochemistry. TSCC and paired adjacent tissue specimens from enrolled patients were acquired from the Pathological Department of the aforementioned hospital; the samples were paraffin-embedded. Then, immunohistochemistry was performed to assess ITGA7 and CD133 expression in tumor and paired adjacent normal tissues. Briefly, paraffin-embedded tissue sections (4- $\mu \mathrm{m}$ thick) were deparaffinized with xylene and rehydrated with ethanol. Heat-induced antigen retrieval was subsequently conducted in $0.01 \mathrm{~mol} / 1$ sodium citrate buffer $(\mathrm{pH}$ 6.0) using a microwave, and endogenous peroxidase activity was inhibited with freshly prepared $3 \% \mathrm{H}_{2} \mathrm{O}_{2}$. Following blocking using $1.5 \%$ normal goat serum (Shanghai Yeasen Biotechnology Co., Ltd.) at $37^{\circ} \mathrm{C}$ for $20 \mathrm{~min}$, the sections were incubated at $4^{\circ} \mathrm{C}$ overnight with rabbit polyclonal ITGA7 antibody (1:200; cat. no. ab203254; Abcam) and rabbit polyclonal CD133 antibody (1:100; cat. no. ab19898; Abcam). The next day, tissue sections were incubated with horseradish peroxidase-conjugated goat anti-rabbit immunoglobulin $\mathrm{G}$ antibody (diluted 1:1,000 in 3\% bovine serum albumin; cat. no. ab6721; Abcam) at $37^{\circ} \mathrm{C}$ for $90 \mathrm{~min}$. Subsequently, 3,3'-diaminobenzidine and hematoxylin were applied for staining of the sections, which were then sealed with neutral tree gum. ITGA7 and CD133 expression was evaluated under a light microscope.

Assessment of ITGA7 and CD133 expression in TSCC and paired adjacent tissues. ITGA7 and CD133 expression in TSCC and paired adjacent tissues was assessed by scores based on the average intensity and percentage of positively stained cells, as described previously (14). The intensity scores were graded as follows: 0, no staining; 1, weak staining, light yellow; 2, moderate staining, yellow brown; and 3, strong staining, brown. The percentage of stained cells was scored as: $0,0 \% ; 1,1-25 \% ; 2,26-50 \% ; 3,51-75 \%$; and $4,76-100 \%$ positive cells. The total score was calculated by multiplying the density score and the percentage score; high expression was defined as a total score of $\geq 3$, while low expression was defined as a total score of $<3$.

Cell sources and culture. Four human TSCC cell lines and one normal human oral keratinocyte cell line were purchased through a third agent company (Shanghai QeeJen Bio-Tech Co., Ltd). In detail, the CAL-27 cell line was purchased from Deutsche Sammlung von Mikroorganismen und Zellkulturen $\mathrm{GmbH}$, the SCC-9 cell line was purchased from American Type Culture Collection, the HSC-4 cell line was purchased from Japanese Collection of Research Bioresources Cell Bank, the SCC-25 cell line was purchased from Cell Bank of Type Culture Collection of Chinese Academy of Sciences, and the HOK cell line was purchased from ScienCell Research Laboratories, Inc.

The CAL-27 and SCC-9 cell lines were cultured in 90\% RPMI-1640 medium (Gibco; Thermo Fisher Scientific, Inc.) with $10 \%$ fetal bovine serum (FBS; Gibco; Thermo Fisher Scientific, Inc.). The HSC-4 cell line was cultured in $90 \%$ Eagle's minimal essential medium (MEM; Gibco; Thermo Fisher Scientific, Inc.) with $10 \%$ FBS. The SCC-25 cell line was cultured in 90\% Dulbecco's modified Eagle's medium/Ham's F12 medium (DMEM/F12; Gibco; Thermo Fisher Scientific, Inc.) with $10 \%$ FBS. The HOK cell line was cultured in $90 \%$ oral keratinocyte medium (ScienCell Research Laboratories, Inc.) with $10 \% \mathrm{FBS}$. All the cells were cultured in incubators at $37^{\circ} \mathrm{C}$ under $95 \%$ air and $5 \% \mathrm{CO}_{2}$ conditions.

ITGA7 and CD133 expression in TSCC and normal cell lines. qPCR, western blotting and immunofluorescence were used for the detection of the mRNA and protein expression levels 
of ITGA7, and western blotting and immunofluorescence were used for the detection of the expression levels of CD133, in the human TSCC cell lines CAL-27, SCC-9, HSC-4 and SCC-25 and the normal human oral keratinocyte cell line HOK (as a normal control).

Lentivirus construction and transduction into CAL-27 and HSC-4 cells. Control short hairpin RNA (shRNA) and ITGA7-targeting shRNA shuttle plasmids were constructed using the pGLV-U6 vector (Shanghai GenePharma Co., Ltd.), and transfected into 293T cells (American Type Culture Collection) together with envelope plasmids (Shanghai GenePharma Co., Ltd.) and packaging plasmids (Shanghai GenePharma Co., Ltd.) using HilyMax (Dojindo Molecular Technologies, Inc.). The cell supernatant was obtained at 48 and $72 \mathrm{~h}$ post-transfection. Following purification and concentrating, the corresponding lentiviruses were collected. The sequences used for the knockdown experiments were as follows: ITGA7 shRNA, forward 5'-CACCGCTGCCCACTC TACAGCTTTTCGAAAAAAGCTGTAGAGTGGGCAGC-3' and reverse, 5'-AAAAGCTGCCCACTCTACAGCTTTTTT CGAAAAGCTGTAGAGTGGGCAGC-3'; control shRNA, forward 5'-CACCGTTCTCCGAACGTGTCACGTCGAAAC GTGACACGTTCGGAGAA-3' and reverse 5'-AAAATTCTC CGAACGTGTCACGTTTCGACGTGACACGTTCGGAGA AC-3'. Subsequently, using a multiplicity of infection of 10 , control shRNA and ITGA7 shRNA lentiviruses were added to the medium for the transduction of CAL-27 and HSC-4 cells with $6 \mu \mathrm{g} / \mathrm{ml}$ polybrene (Sigma-Aldrich; Merck KGaA) for $24 \mathrm{~h}$, followed by the addition of fresh complete medium for another $48 \mathrm{~h}$. Then, cells were cultured with $8 \mu \mathrm{g} / \mathrm{ml}$ puromycin (Thermo Fisher Scientific, Inc.) for 7 days to construct stably transduced CAL-27 and HSC-4 cells. Cells transduced with the control shRNA lentivirus were termed as the negative control (NC) group, while the cells transduced with the ITGA7shRNA lentivirus were termed as the ITGA7(-) group.

ITGA7 expression in the NC and ITGA7(-) groups. Following the construction of stably transduced CAL-27 and HSC-4 cells, ITGA7 expression was detected by RT-qPCR, western blotting and flow cytometry in the NC and ITGA7(-) groups, in order to determine successful transduction.

Effects of ITGA7 knockdown on the proliferation and apoptosis of CAL-27 and HSC-4 cells. In the NC and ITGA7(-) groups of stably transduced CAL-27 and HSC-4 cells, the apoptotic rate was detected using a fluorescein isothiocyanate (FITC) Annexin-V Apoptosis Detection kit II (BD Biosciences). Expression levels of the cell apoptotic markers cleaved (C)-Caspase 3 and $\mathrm{Bcl}-2$ were detected via western blotting. In addition, an equal quantity of cells of the $\mathrm{NC}$ and ITGA7(-) groups were cultured for $72 \mathrm{~h}$, and the proliferative ability was detected at $0,24,48$ and $72 \mathrm{~h}$ via a Cell Counting Kit-8 (CCK-8) assay (Sigma-Aldrich; Merck KGaA).

Effects of ITGA7 knockdown on CSC markers of CAL-27 and HSC-4 cells. In the NC and ITGA7(-) groups of stably transduced CAL-27 and HSC-4 cells, the CSC markers cluster of differentiation (CD)24, CD44 and CD133 were detected by RT-qPCR and western blotting. In addition, ITGA-positive cells, as well as ITGA7-negative cells, were isolated by flow cytometry, and the protein expression levels of ITGA7, CD24, CD44 and CD133 were detected by western blotting.

Effects of ITGA7 knockdown on drug resistance to cisplatin in CAL-27 and HSC-4 cells. Cisplatin (5 $\mu \mathrm{M}$; Sigma-Aldrich; Merck KGaA) was applied to the NC and ITGA7(-) groups of stably transduced CAL-27 and HSC-4 cells for $24 \mathrm{~h}$. Then, the drug resistance ability was assessed by detecting cell viability and apoptosis. Briefly, cell viability was detected via a CCK-8 assay and the relative cell viability of the ITGA7 (-) group was calculated according to the reference values of the NC group. The rate of cell apoptosis was determined using an FITC Annexin-V Apoptosis Detection Kit II.

Effects of ITGA7 knockdown on the sphere formation ability of CAL-27 and HSC-4 cells. The sphere formation ability of the NC and ITGA7(-) groups of stably transduced CAL-27 and HSC-4 cells was analyzed by a sphere formation assay. Briefly, transduced CAL-27 and HSC-4 cells were cultured in DMEM/F12 medium supplemented with 2\% B27 (Gibco; Thermo Fisher Scientific, Inc.), $20 \mathrm{ng} / \mathrm{ml}$ epidermal growth factor (EGF; Sigma-Aldrich; Merck KGaA), $20 \mathrm{ng} / \mathrm{ml}$ basic fibroblast growth factor (bFGF; Gibco; Thermo Fisher Scientific, Inc.) and $4 \mu \mathrm{g} / \mathrm{ml}$ heparin (Sigma-Aldrich; Merck $\mathrm{KGaA}$ ) for 10 days; spheres of a diameter $>50 \mu \mathrm{m}$ were counted under a light microscope (Olympus Corporation). The sphere formation ability was calculated by dividing the number of these spheres by the total number of seeded cells (200) x1,000. In addition, the sphere formation assay was conducted using the extreme limiting dilution method.CAL-27 and HSC-4 cells were cultured in DMEM/F12 medium supplemented with $2 \%$ B27, 20 ng/ml EGF (Sigma-Aldrich; Merck KGaA), 20 ng/ml $\mathrm{bFGF}$ and $4 \mu \mathrm{g} / \mathrm{ml}$ heparin (Sigma-Aldrich; Merck KGaA) for 10 days in a 24 well-plate at densities of $1,000,100$ or 10 cells per well. Subsequently, spheres with a diameter $>50 \mu \mathrm{m}$ were counted under a light microscope (Olympus Corporation) and the sphere formation ability was calculated using an extreme limiting dilution analysis software (http://bioinf.wehi.edu. au/software/elda/) (15).

Expression of ITGA7 in CAL-27 and HSC-4 CSCs. CAL-27 and HSC-4 CSCs were generated by establishing drug-resistant cells, followed by detection with a sphere formation assay (16). Briefly, $5 \mu \mathrm{M}$ cisplatin was applied to CAL-27 and HSC-4 cells for $72 \mathrm{~h}$, and then cisplatin-free medium was applied to cells for another $72 \mathrm{~h}$; these processes were repeated until no effect of cisplatin on cell viability/proliferation was observed by the CCK-8 assay. A sphere formation assay was performed as aforementioned, and spheres of CAL-27 and HSC-4 CSCs were isolated by centrifugation ( $300 \mathrm{x} \mathrm{g}$ at room temperature for $3 \mathrm{~min}$ ). To determine the successful establishment of CSCs, the expression levels of CSC markers CD24, CD44 and CD133, as well as ITGA7, were detected by RT-qPCR and western blotting in CAL-27/HSC-4 CSCs and normal parental CAL-27/HSC-4 cells.

$R T$ - $q P C R$. TRIzol ${ }^{\circledR}$ reagent (Thermo Fisher Scientific, Inc.) was used for the extraction of total RNA. The ReverTra Ace ${ }^{\circledR}$ qPCR RT kit (Toyobo Life Science) was used to transcribe $1 \mu \mathrm{g}$ RNA into cDNA. SYBR ${ }^{\circledR}$ Green Realtime PCR Master Mix (Toyobo 
Table I. Primers used in quantitative PCR.

\begin{tabular}{lll}
\hline Gene & \multicolumn{1}{c}{ Forward primer (5'-3') } & \multicolumn{1}{c}{ Reverse primer (5'-3') } \\
\hline ITGA7 & GCCACTCTGCCTGTCCAATG & GGAGGTGCTAAGGATGAGGTAGA \\
CD24 & GCTCCTACCCACGCAGATTT & CACGAAGAGACTGGCTGTTGA \\
CD44 & ACATCCTCACATCCAACACCTC & CCTCCTGAAGTGCTGCTCCT \\
CD133 & GCTGCTTGTGGAATAGACAGAATG & GAAGGACTCGTTGCTGGTGAAT \\
GAPDH & GAGTCCACTGGCGTCTTCAC & ATCTTGAGGCTGTTGTCATACTTCT
\end{tabular}

ITGA7, integrin $\alpha 7$.

Table II. Antibodies used in western blotting.

A, Primary antibodies

\begin{tabular}{llcr}
\hline Target & Catalog no. & Company & Dilution \\
\hline ITGA7 & ab203254 & Abcam r & $1: 2,000$ \\
Caspase-3 & $\# 14220$ & Cell Signaling Technology, Inc. & $1: 1,000$ \\
Cleaved caspase-3 & $\# 9661$ & Cell Signaling Technology, Inc. \\
Bcl-2 & ab32124 & Abcam & Abcam \\
CD24 & ab179821,000 & $1: 1,000$ \\
CD44 & ab51037 & Abcam \\
CD133 & ab19898 & Abcam \\
GAPDH & $\# 2118$ & Cell Signaling Technology, Inc. \\
\hline
\end{tabular}

B, Secondary antibody

\begin{tabular}{llll}
\hline Target & Catalog no. & Company & Dilution \\
\hline HRP-conjugated goat anti-rabbit IgG & ab205718 & Abcam & $1: 2,000$ \\
\hline
\end{tabular}

ITGA7, integrin $\alpha 7$; HRP, horseradish peroxidase; IgG, immunoglobulin G.

Life Science) was used for qPCR, which was performed as follows: $95^{\circ} \mathrm{C}$ for $5 \mathrm{~min}$, followed by 40 cycles of $95^{\circ} \mathrm{C}$ for $5 \mathrm{sec}$ and $61^{\circ} \mathrm{C}$ for $30 \mathrm{sec}$. The sequences of primers employed for qPCR are listed in Table I. Relative fold changes in mRNA expression were calculated using the $2^{-\Delta \Delta \mathrm{Cq}}$ method $(17,18)$. GAPDH was used as the internal reference.

Western blotting. Radioimmunoprecipitation assay buffer (Thermo Fisher Scientific, Inc.) was used for the extraction of total protein, and a Pierce ${ }^{\mathrm{TM}}$ BCA Protein Assay kit (Thermo Fisher Scientific, Inc.) was used to determine protein concentration; a standard curve was used for adjustments. The protein sample $(20 \mu \mathrm{g})$ was fractionated via a NuPAGE ${ }^{\mathrm{TM}} 4-12 \%$ Bis-Tris Protein Gel (Invitrogen; Thermo Fisher Scientific, Inc.), and then transferred to nitrocellulose filter membranes (EMD Millipore). Subsequently, the membranes were blocked using 5\% skim milk for $2 \mathrm{~h}$ at $37^{\circ} \mathrm{C}$, and were incubated with primary antibodies overnight at $4^{\circ} \mathrm{C}$. After the secondary antibody was applied for $1 \mathrm{~h}$ at room temperature, Pierce ${ }^{\mathrm{TM}}$ Enhanced Chemiluminescence Plus Western Blotting Substrate (Thermo Fisher Scientific, Inc.) was used for the visualization of bands, which were then exposed using X-ray film (Kodak). GAPDH was used as the internal reference. The detailed information regarding the antibodies employed for western blot analysis are listed in Table II.

Immunofluorescence. Cells were fixed with $4 \%$ paraformaldehyde for $10 \mathrm{~min}$ at room temperature, permeabilized with $0.2 \%$ Triton X-100 for 5 min and blocked with PBS containing $2 \%$ BSA for $1 \mathrm{~h}$ at room temperature. Cells were then incubated with primary antibodies targeting ITGA7 (1:200; cast. no. ab203254; Abcam) and anti-CD133 (1:100; cat. no. ab19898; Abcam) overnight at $4^{\circ} \mathrm{C}$. Following three washes with PBS, cells were incubated with an FITC-conjugated goat anti-rabbit immunoglobulin $\mathrm{G}$ antibody (1:1,000; cat. no. ab6717; Abcam) for $1 \mathrm{~h}$ at $37^{\circ} \mathrm{C}$. The nuclei were counterstained with DAPI. Images were obtained using a BX41 fluorescence microscope (Olympus Corporation) at x400 magnification.

Flow cytometry. Cells were harvested and washed with PBS, and then incubated with rabbit polyclonal antibody against 
ITGA7 (1:50; cat. no. ab203254; Abcam) in incubation buffer (PBS) for $1 \mathrm{~h}$ at $4^{\circ} \mathrm{C}$. Then, cells were washed with PBS and incubated with a FITC-conjugated goat anti-rabbit immunoglobulin $\mathrm{G}$ antibody (1:1,000; cat. no. ab6717; Abcam) for $1 \mathrm{~h}$ at $4^{\circ} \mathrm{C}$ in the dark. Subsequently, the cells were washed again, resuspended in FACS buffer (PBS) and analyzed by flow cytometry (Flowjo Vision 7.6; BD Biosciences) using a FACS Canto II flow cytometer (BD Biosciences).

Statistical analysis. Statistical analysis was performed using SPSS 22.0 software (IBM Corp.) and GraphPad Prism 7.00 (GraphPad Software Inc.). Normal distributed continuous variables were presented as the mean \pm standard deviation, and categorized variables were presented as a percentage. Comparisons of ITGA7 expression between tumor and paired adjacent tissues were conducted via a McNemar test. Associations between ITGA7 expression and patient characteristics were determined by a $\chi^{2}$ or Wilcoxon rank sum test. Differences between the OS of patients with low and high ITGA7 expression were determined via Kaplan-Meier analysis, followed a log-rank test. Univariate and multivariate Cox proportional hazards regression analyses were performed to determine the factors affecting OS. Comparisons among groups in cell experiments were conducted with one-way ANOVA followed by Dunnett's multiple comparisons test. Comparisons between two groups in cell experiments were performed with unpaired parametric t-test. $\mathrm{P}<0.05$ was considered to indicate a statistically significant difference.

\section{Results}

Baseline characteristics in patients with TSCC. A total of 60 patients with TSCC were enrolled with a mean age of 55.2 \pm 10.5 years, comprising 44 males and 16 females (Table III). Regarding pathological grade, the number of patients with TSCC in grade $(\mathrm{G}) 1, \mathrm{G} 2$ and $\mathrm{G} 3$ were $8(13.3 \%), 42(70.0 \%)$ and $10(16.7 \%)$, respectively. There were $10(16.7 \%), 24(40.0 \%), 24(40.0 \%)$ and $2(3.3 \%)$ patients with TSCC of TNM stage I, II, III and IV, respectively. The detailed information of other clinicopathological characteristics of the patients is presented in Table III.

Comparison of ITGA7 expression between tumor tissue and paired adjacent tissue. Immunohistochemistry was conducted for the detection of ITGA7 expression in tumor and paired adjacent tissues; representative images of high and low ITGA7 staining are presented in Fig. 1A. The results from the immunohistochemistry analysis demonstrated that the protein expression levels of ITGA7 were increased in the tumor tissues compared with paired adjacent tissues ( $\mathrm{P}=0.019$; Fig. 1B). Similar results were obtained by RT-qPCR analysis for the ITGA7 mRNA expression levels ( $\mathrm{P}<0.001$; Fig. 1C). In addition, the expression of the CSC marker CD133 was detected by immunohistochemistry in the patient tissues (Fig. S1A). The results revealed that $\mathrm{CD} 133$ expression was upregulated in tumor tissues compared with adjacent normal tissues $(\mathrm{P}=0.048$; Fig. S1B).

Association between ITGA7 expression and the clinicopathological characteristics of patients with TSCC. Increased
Table III. Clinical characteristics of patients with TSCC.
Characteristics

Age (years)

Gender (male/female)

Pathological grade, n (\%)

G1

$\mathrm{G} 2$

G3

T stage, n (\%)

T1

$\mathrm{T} 2$

T3

$\mathrm{N}$ stage, $\mathrm{n}(\%)$

NO

$\mathrm{N} 1$

$\mathrm{N} 2$

TNM stage, n (\%) I

II

III

IV
TSCC patients $(\mathrm{n}=60)$

$55.2 \pm 10.5$

$44 / 16$

$8(13.3)$

$42(70.0)$

10 (16.7)

$12(20.0)$

$30(50.0)$

$18(30.0)$

38 (63.3)

10 (16.7)

$24(40.0)$

$24(40.0)$

2 (3.3)
Data are presented as mean \pm standard deviation or count (percentage). TSCC, tongue squamous cell carcinoma; TNM, tumor-node-metastasis.

protein expression of ITGA7 was associated with advanced pathological grade $(\mathrm{P}=0.021)$, and higher $\mathrm{N}(\mathrm{P}=0.011)$ and TNM $(\mathrm{P}=0.005)$ stages. However, no association between ITGA7 protein expression and age $(\mathrm{P}=0.465$ and $0.876)$ or gender $(\mathrm{P}=0.876)$ was observed in patients with TSCC (Table IV). In addition, high ITGA7 mRNA expression was associated with advanced pathological grade $(\mathrm{P}=0.003)$, increased T stage $(\mathrm{P}=0.002)$ and higher TNM stage $(\mathrm{P}=0.010)$ in patients with TSCC (Table IV).

Association between ITGA7 expression and the OS of patients with TSCC. Kaplan-Meier analysis revealed that the OS was shorter in patients with high ITGA7 protein expression levels [meanOS: 36.2 months, 95\% confidenceinterval(CI):31.24-41.1 months] compared with those possessing low ITGA7 protein expression (mean OS: 48.1 months, 95\% CI: 44.9-51.3 months; $\mathrm{P}=0.013$; Fig. 2A). In addition, a similar trend was reported for patients with high ITGA7 mRNA expression (mean OS: 37.2 months, 95\% CI: 31.6-42.9 months) compared with those with low ITGA7 mRNA expression (mean OS: 48.2 months, 95\% CI: 45.3-51.3 months; P=0.007; Fig. 2B).

Factors affecting the OS of patients with TSCC. Univariate Cox proportional hazards regression model analysis was used to evaluate the effect of all clinicopathological factors on the OS of patients with TSCC. The results revealed that high ITGA7 protein $(\mathrm{P}=0.024)$ and mRNA expression $(\mathrm{P}=0.015)$, higher pathological grade $(\mathrm{P}=0.049)$, advanced $\mathrm{T}$ stage $(\mathrm{P}=0.012)$, higher $\mathrm{N}$ stage $(\mathrm{P}=0.001)$, as well as advanced TNM 

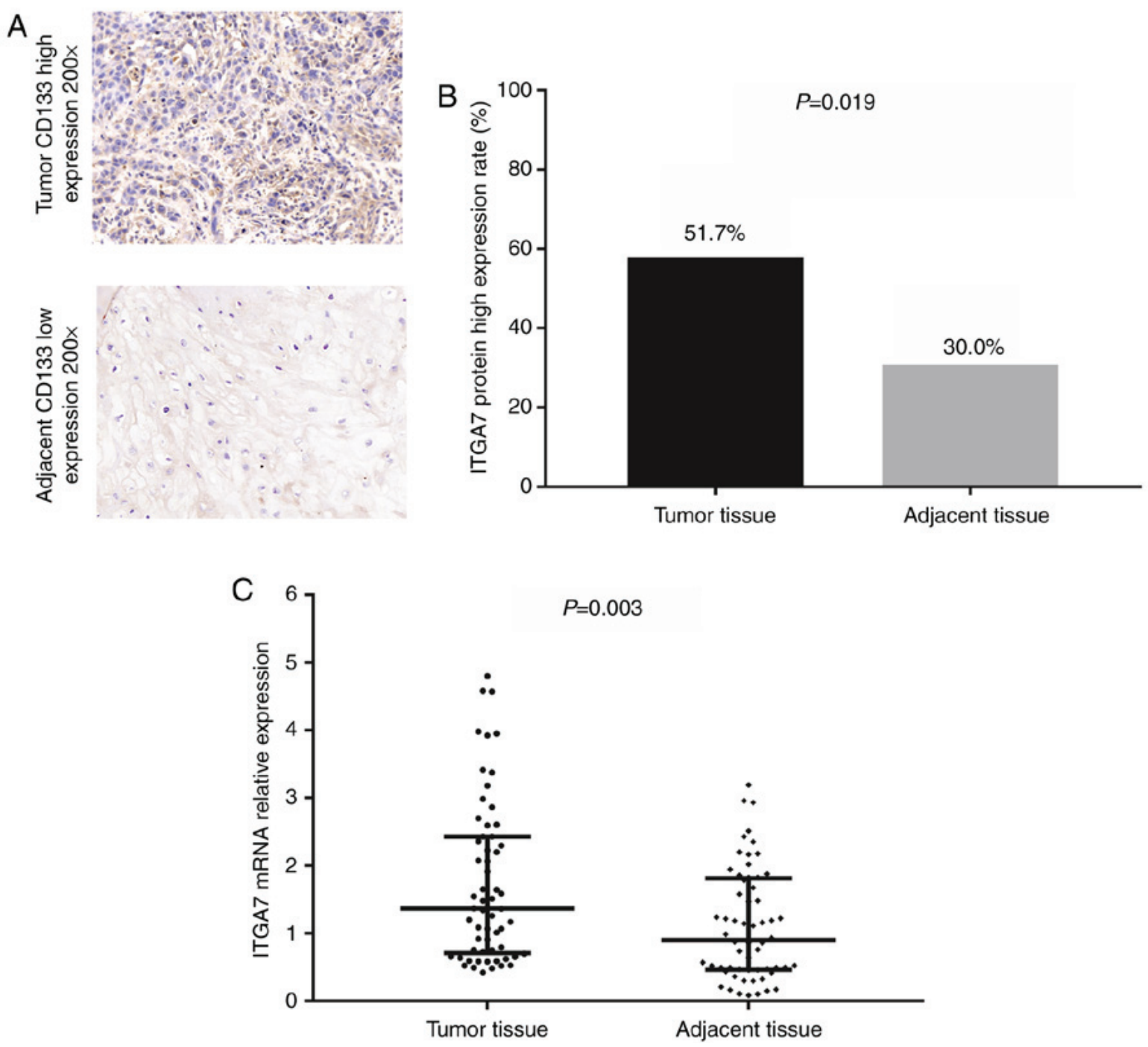

Figure 1. ITGA7 protein and mRNA expression in tongue squamous cell carcinoma and adjacent normal tissues. (A) Representative images of ITGA7 high or low protein expression staining (magnification, x200). (B) Quantification of tissues with high ITGA7 protein expression. (C) mRNA expression levels of ITGA7 in tumor and paired adjacent tissues. ITGA7, integrin $\alpha 7$.

stage $(\mathrm{P}<0.001)$ were associated with poorer OS in patients with TSCC (Table V). However, when multivariate Cox analysis was performed, only TNM stage could independently predict OS, while high ITGA7 expression (protein or mRNA) was not an independent factor for predicting shorter OS in patients with TSCC. This suggested that ITGA7 may be indirectly associated with poor prognosis by affecting tumor size, pathological grade or lymph node metastasis in patients with TSCC.

Comparison of ITGA7 expression in TSCC and normal human oral keratinocyte cell lines. In order to investigate the function of ITGA7 in TSCC cells, in vitro experiments were performed. Firstly, the expression of ITGA7 was detected in several established TSCC cell lines and a normal human oral keratinocyte cell line. Compared to the normal HOK cells, both ITGA7 mRNA (Fig. 3A) and protein (Fig. 3B) expression levels were increased in the human TSCC cell lines CAL-27, SCC-9, HSC-4 and SCC-25.

ITGA7 knockdown in CAL-27 and HSC-4 cells. In order to investigate the underlying mechanism of ITGA7 in CAL-27 and HSC-4 cells, control NC shRNA and ITGA7 shRNA lentiviruses were constructed and used to transduce these cell lines, hence generating the NC and ITGA7(-) cell groups, respectively. In CAL-27 cells, the mRNA ( $\mathrm{P}<0.001$; Fig. 4A) and protein (Fig. 4B) expression levels of ITGA7 were downregulated in the ITGA7(-) group compared with the NC group. Additionally, a similar trend of ITGA7 expression at the mRNA ( $<<0.001$; Fig. 4C) and protein (Fig. 4D) levels was observed between the ITGA7(-) and NC groups of HSC-4 cells. These findings suggested the successful construction of stably transduced ITGA7-silenced TSCC cell lines. In addition, the results of flow cytometry demonstrated that the percentage of ITGA7+ cells was decreased in the ITGA7(-) group compared with the NC group, for both the CAL-27 and HSC-4 cell lines $(\mathrm{P}<0.01$; Fig. S2A-D).

Effects of ITGA7 knockdown on the proliferation and apoptosis of CAL-27 and HSC-4 cells. The present study investigated the effects of ITGA7 knockdown on the proliferation and apoptosis of CAL-27 and HSC-4 cells. A CCK- 8 assay revealed that cell proliferation was decreased in the ITGA7(-) 
Table IV. Association of ITGA7 expression with patients' clinical characteristics.

\begin{tabular}{|c|c|c|c|c|c|c|c|}
\hline \multirow[b]{2}{*}{ Parameters } & \multirow[b]{2}{*}{$\mathrm{n}$} & \multicolumn{2}{|c|}{ ITGA7 protein expression } & \multirow[b]{2}{*}{ P-value } & \multicolumn{2}{|c|}{ ITGA7 mRNA expression } & \multirow[b]{2}{*}{ P-value } \\
\hline & & Low & High & & Low & High & \\
\hline Age, n (\%) & & & & 0.465 & & & 0.584 \\
\hline$<60$ years & 40 & $18(45.0)$ & $22(55.0)$ & & $21(52.5)$ & $19(47.5)$ & \\
\hline$\geq 60$ years & 20 & $11(55.0)$ & $9(45.0)$ & & $9(45.0)$ & $11(55.0)$ & \\
\hline Gender, n (\%) & & & & 0.876 & & & 0.243 \\
\hline Male & 44 & $21(47.8)$ & $23(52.2)$ & & $20(45.5)$ & $24(54.5)$ & \\
\hline Female & 16 & $8(50.0)$ & $8(50.0)$ & & $10(62.5)$ & $6(37.5)$ & \\
\hline Pathological grade, n (\%) & & & & 0.021 & & & 0.003 \\
\hline G1 & 8 & $7(87.5)$ & $1(12.5)$ & & $8(100.0)$ & $0(0.0)$ & \\
\hline $\mathrm{G} 2$ & 42 & $19(45.2)$ & $23(54.8)$ & & $20(47.6)$ & $22(52.4)$ & \\
\hline G3 & 10 & $3(30.0)$ & $7(70.0)$ & & $2(20.0)$ & $8(80.0)$ & \\
\hline T stage, $\mathrm{n}(\%)$ & & & & 0.073 & & & 0.002 \\
\hline $\mathrm{T} 1$ & 12 & $8(66.7)$ & $4(33.3)$ & & $9(75.0)$ & $3(25.0)$ & \\
\hline $\mathrm{T} 2$ & 30 & $15(50.0)$ & $15(50.0)$ & & $18(60.0)$ & $12(40.0)$ & \\
\hline $\mathrm{T} 3$ & 18 & $6(33.3)$ & $12(66.7)$ & & $3(16.7)$ & $15(83.3)$ & \\
\hline N stage, n (\%) & & & & 0.011 & & & 0.064 \\
\hline No & 38 & $23(60.5)$ & $15(39.5)$ & & $23(60.5)$ & $15(39.5)$ & \\
\hline N1 & 20 & $6(30.0)$ & $14(70.0)$ & & $7(35.0)$ & $13(65.0)$ & \\
\hline $\mathrm{N} 2$ & 2 & $0(0.0)$ & $2(100.0)$ & & $0(0.0)$ & $2(100.0)$ & \\
\hline TNM stage, n (\%) & & & & 0.005 & & & 0.010 \\
\hline I & 10 & $8(80.0)$ & $2(20.0)$ & & $8(80.0)$ & $2(20.0)$ & \\
\hline II & 24 & $13(54.2)$ & $11(45.8)$ & & $15(62.5)$ & $9(37.5)$ & \\
\hline III & 24 & $8(33.3)$ & $16(66.7)$ & & $7(29.2)$ & $17(70.8)$ & \\
\hline IV & 2 & $0(0.0)$ & $2(100.0)$ & & $0(0.0)$ & $2(100.0)$ & \\
\hline
\end{tabular}

The patients were divided into high or low ITGA7 mRNA expression groups based on the median value of ITGA7 mRNA relative expression. Comparison between two groups was determined by Chi-square or Wilcoxon rank sum test. Significant P-values are denoted in bold font. ITGA7, integrin $\alpha 7$; G, grade; T, tumor; $\mathrm{N}$, node; TNM, tumor-node-metastasis.
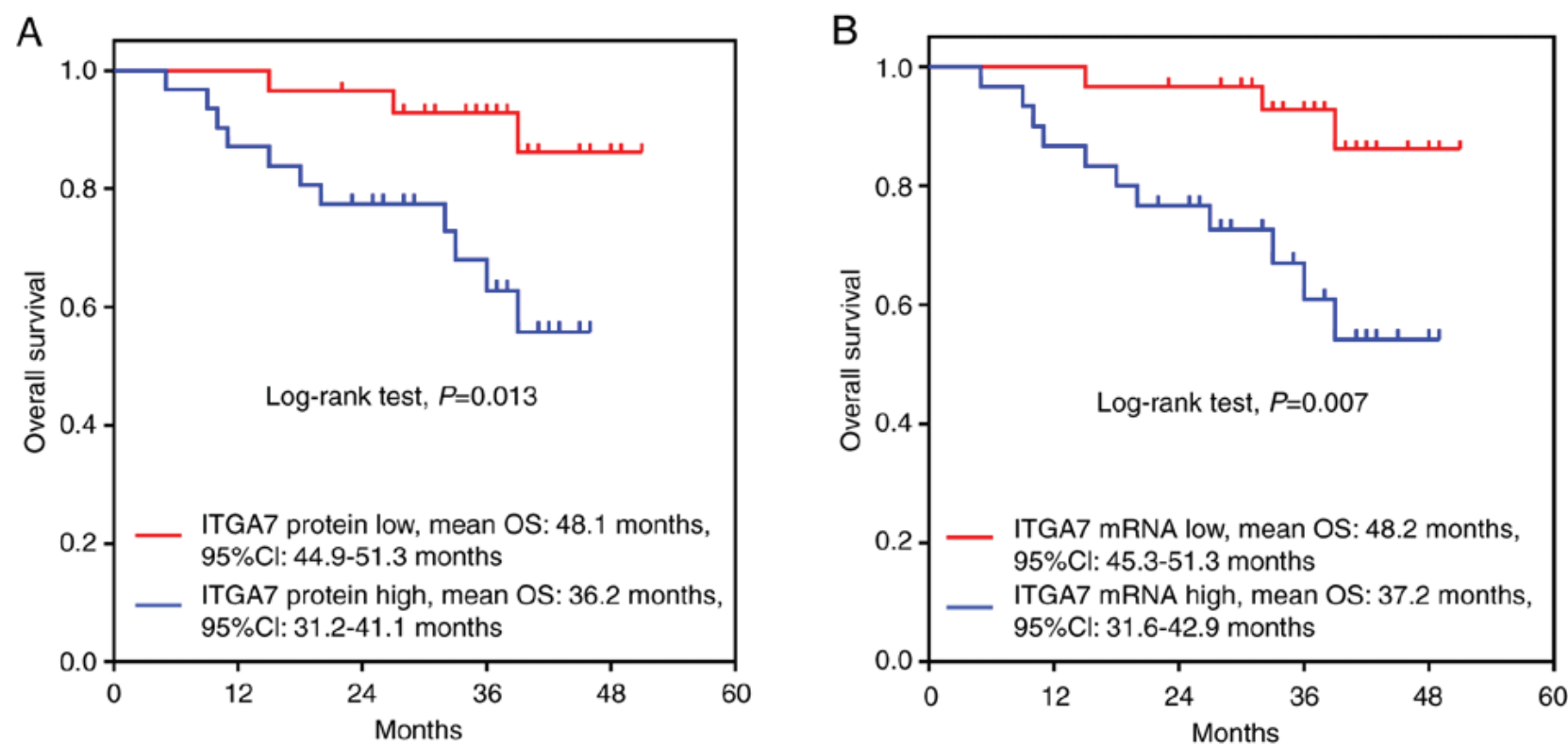

Figure 2. High ITGA7 expression is associated with poor OS in patients with TSCC. (A) Association of ITGA7 protein expression with OS in patients with TSCC. (B) Association of ITGA7 mRNA expression with OS in patients with TSCC. ITGA7, integrin $\alpha 7$; OS, overall survival; TSCC, tongue squamous cell carcinoma. 
Table V. Univariate and multivariate Cox proportional hazards regression model analysis of factors affecting overall survival.

A, Univariate analysis

Cox proportional hazards regression model

\begin{tabular}{lrrrr} 
& & & \multicolumn{2}{c}{$95 \%$ CI } \\
\cline { 3 - 5 } Parameters & P-value & HR & Lower & Higher \\
\hline ITGA7 protein high expression & $\mathbf{0 . 0 2 4}$ & 4.384 & 1.219 & 15.769 \\
ITGA7 mRNA high expression & $\mathbf{0 . 0 1 5}$ & 4.927 & 1.367 & 17.756 \\
Age & 0.637 & 1.011 & 0.966 & 1.059 \\
Gender (male) & 0.102 & 5.460 & 0.713 & 61.790 \\
Higher pathological grade & $\mathbf{0 . 0 4 9}$ & 2.499 & 0.999 & 7.199 \\
Higher T stage & $\mathbf{0 . 0 1 2}$ & 3.030 & 1.276 & 17.216 \\
Higher N stage & $\mathbf{0 . 0 0 1}$ & 5.990 & 2.084 & 51.838 \\
Higher TNM stage & $\mathbf{0 . 0 0 1}$ & 12.764 & 3.143 & \\
\hline
\end{tabular}

B, Multivariate analysis

Cox proportional hazards regression model

\begin{tabular}{lcccr}
\cline { 3 - 4 } & & & \multicolumn{2}{c}{$95 \%$ CI } \\
\cline { 3 - 4 } Parameters & P-value & HR & Lower & Higher \\
\hline ITGA7 protein high expression & 0.643 & 1.416 & 0.326 & 6.140 \\
ITGA7 mRNA high expression & 0.641 & 1.469 & 0.292 & 7.384 \\
Age & 0.908 & 1.073 & 0.326 & 3.528 \\
Gender (male) & 0.462 & 2.236 & 0.262 & 19.069 \\
Higher pathological grade & 0.144 & 3.046 & 0.683 & 13.594 \\
Higher T stage & 0.219 & 0.502 & 0.167 & 1.507 \\
Higher N stage & 0.244 & 0.364 & 0.067 & 1.993 \\
Higher TNM stage & $\mathbf{0 . 0 0 8}$ & 25.675 & 2.305 & 285.942 \\
\hline
\end{tabular}

The patients were divided into high or low ITGA7 mRNA expression groups based on the median value of ITGA7 mRNA relative expression. Factors affecting OS were determined by univariate and multivariate Cox proportional hazards regression analysis. Significant P-values are denoted in bold font. ITGA7, integrin $\alpha 7$; HR, hazards ratio; CI, confidence interval; T, tumor; N, node; TNM, tumor-node-metastasis .

group compared with the $\mathrm{NC}$ group at $48(\mathrm{P}<0.05)$ and 72 $\mathrm{h}(\mathrm{P}<0.01)$ for CAL-27 cells (Fig. 5A), and at $48(\mathrm{P}<0.05)$ and $72 \mathrm{~h}(\mathrm{P}<0.05)$ for HSC-4 cells (Fig. 5E). The rate of cell apoptosis was increased in the ITGA7(-) group compared with the NC group for CAL-27 cells $(\mathrm{P}<0.01$; Fig. 5B and C) and HSC-4 cells $(\mathrm{P}<0.05$; Fig. $5 \mathrm{~F}$ and $\mathrm{G})$. Western blot analysis revealed that the expression of the apoptotic protein marker C-Caspase 3 was increased, but the expression of the anti-apoptotic Bcl-2 was decreased, in the ITGA7(-) group compared with the NC group for CAL-27 cells (Fig. 5D) and HSC-4 cells (Fig. 5H). These findings indicated that ITGA7 knockdown inhibited cell proliferation, but promoted apoptosis in CAL-27 and HSC-4 cells.

Effects of ITGA7 knockdown on regulating common CSC markers in CAL-27 and HSC-4 cells. To investigate the effects of ITGA7 knockdown on the stemness of TSCC cells, the expression of the common CSC markers CD24, CD44 and CD133 was examined in CAL-27 and HSC-4 cells. RT-qPCR and western blotting demonstrated that the mRNA and protein expression of CD24 $(\mathrm{P}<0.001$; Fig. 6A and D) were increased, while that of CD44 $(\mathrm{P}<0.01$; Fig. 6B and D) and CD133 ( $<<0.01$; Fig. 6C and D) were decreased in the ITGA7(-) group compared with the NC group of CAL-27 cells. Additionally, similar trends were observed in the expression of CD24 ( $\mathrm{P}<0.05$; Fig. 6E and H), CD44 ( $\mathrm{P}<0.05$; Fig. 6F and $\mathrm{H})$ and $\mathrm{CD} 133(\mathrm{P}<0.01$; Fig. $6 \mathrm{G}$ and $\mathrm{H})$ between the ITGA7(-)and NC groups of HSC-4 cells. These results suggested that ITGA7 knockdown regulated the expression of common CSC markers in CAL-27 and HSC-4 cells.

In addition, immunofluorescence experiments were performed to detect ITGA7 and CD133 expression in TSCC cell lines and the normal human oral keratinocytes cell line, and the results revealed that ITGA7 and CD133 expression levels were markedly increased in human TSCC cell lines CAL-27, SCC-9, HSC-4 and SCC-25 compared with the 


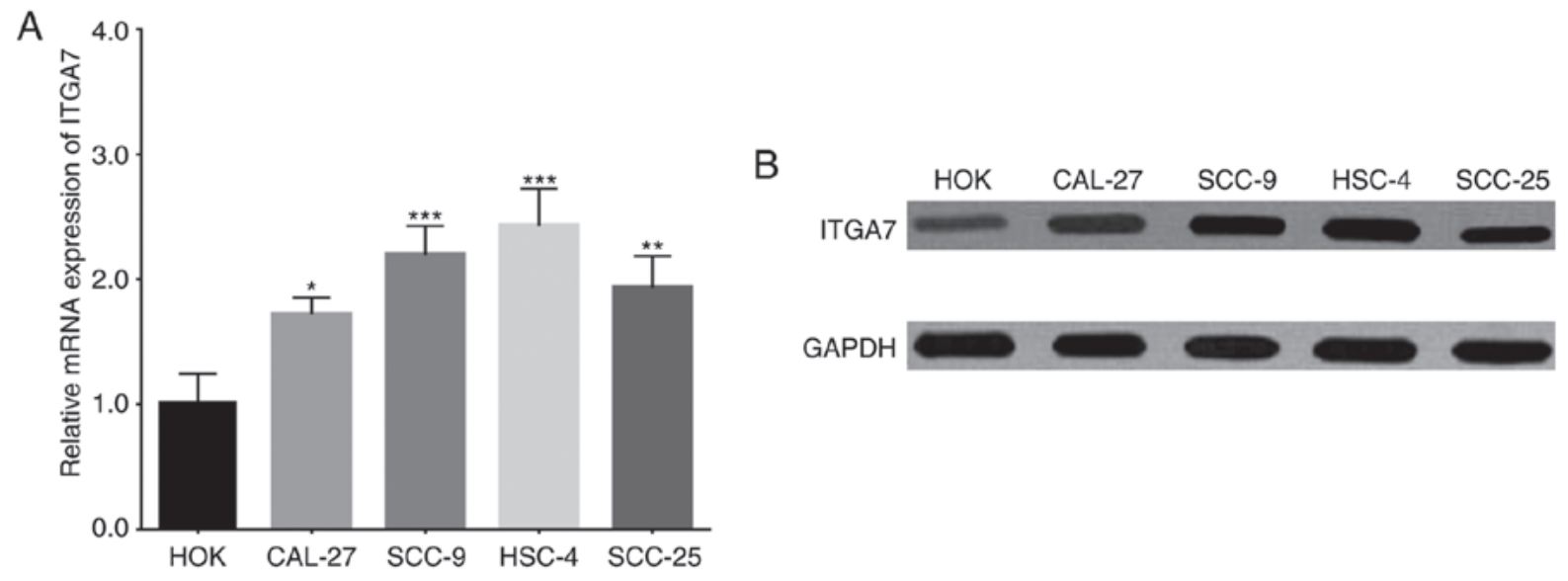

Figure 3. ITGA7 expression is increased in TSCC cell lines compared with normal human oral keratinocytes. (A) mRNA expression levels and (B) protein expression levels of ITGA7 in the human TSCC cell lines CAL-27, SCC-9, HSC-4 and SCC-25 and in the normal human oral keratinocyte cell line HOK. ${ }^{*} \mathrm{P}<0.05,{ }^{* *} \mathrm{P}<0.01$ an ${ }^{* * *} \mathrm{P}<0.001$ compared with HOK. ITGA7, integrin $\alpha 7$; TSCC, tongue squamous cell carcinoma.

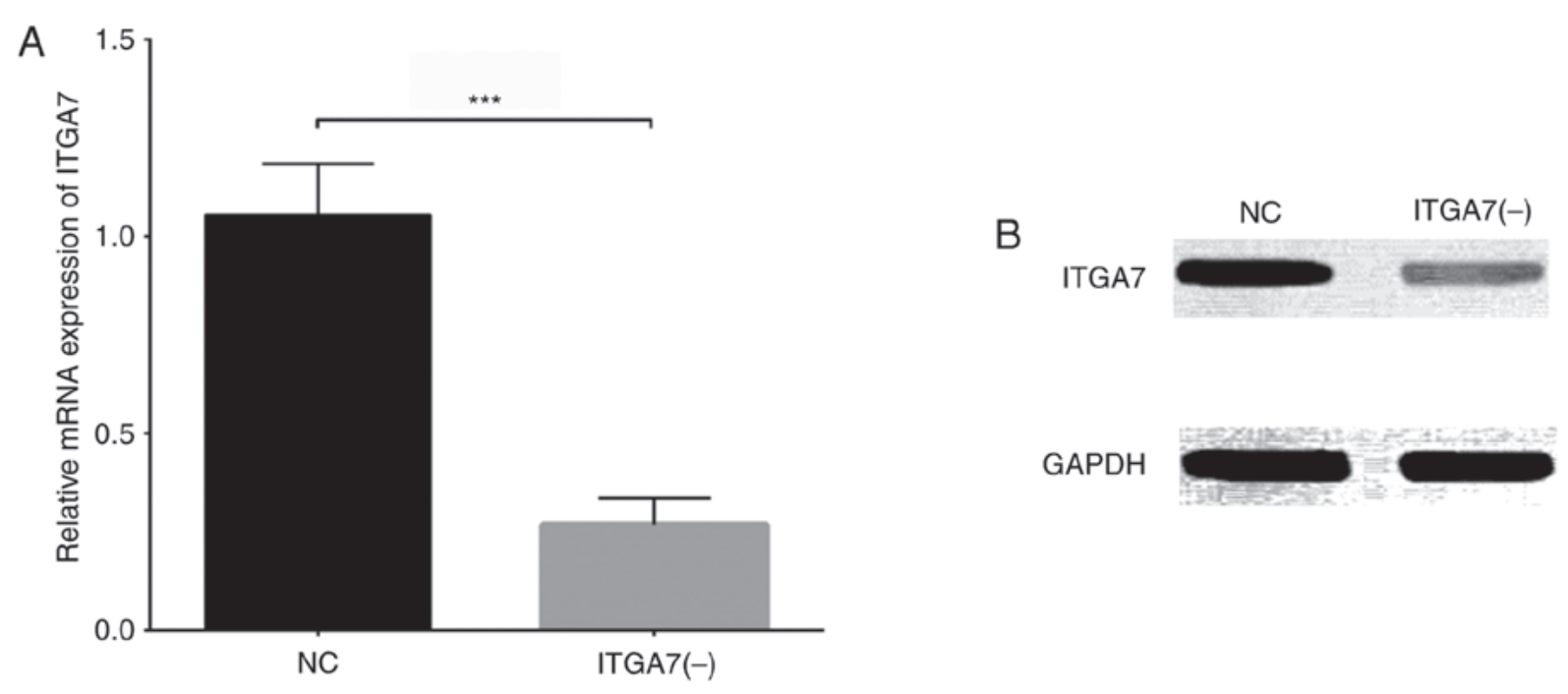

HSC-4

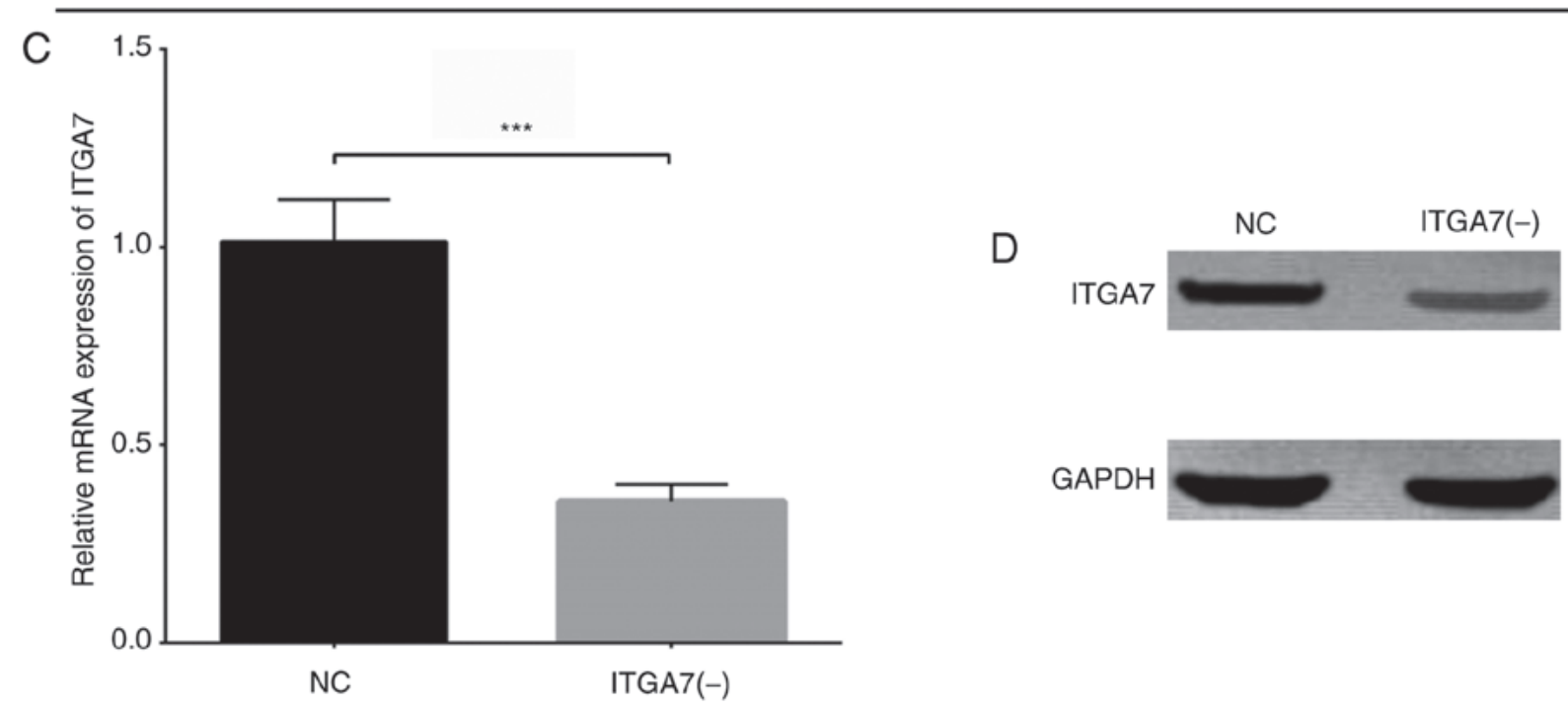

Figure 4. ITGA7 expression in the NC and ITGA7(-) groups. (A) mRNA and (B) protein expression levels of ITGA7 in the ITGA7(-) and NC groups of CAL-27 cells. (C) mRNA and (D) protein expression levels of ITGA7 in the ITGA7(-) and NC groups of HSC-4 cells. ${ }^{* * *} \mathrm{P}<0.001$. ITGA7, integrin $\alpha 7$; NC, negative control. 
CAL-27

A
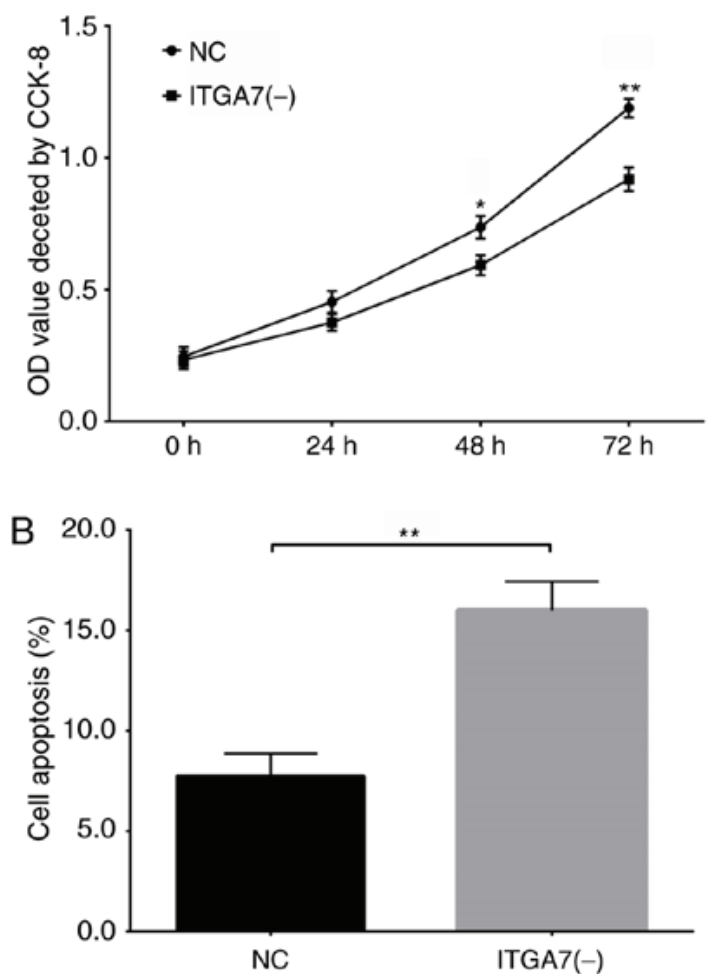

C
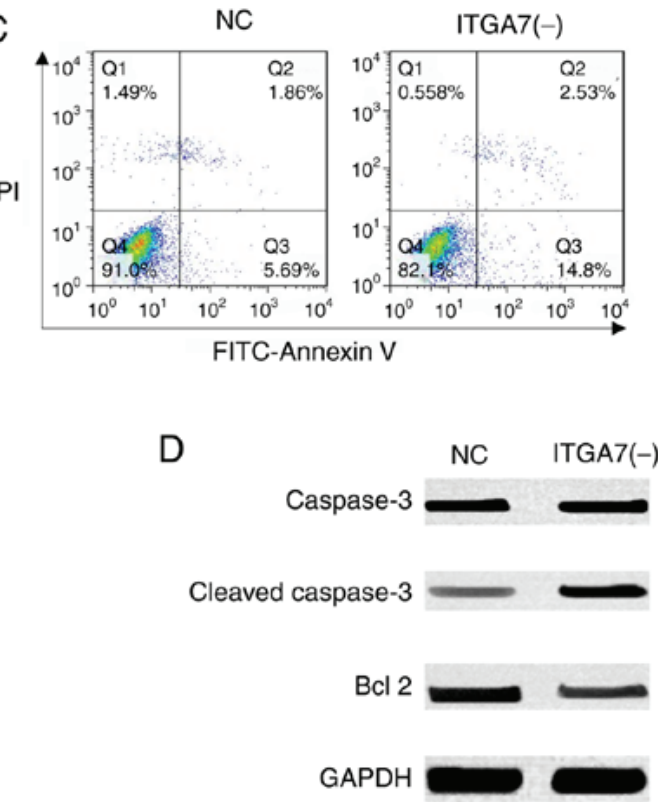
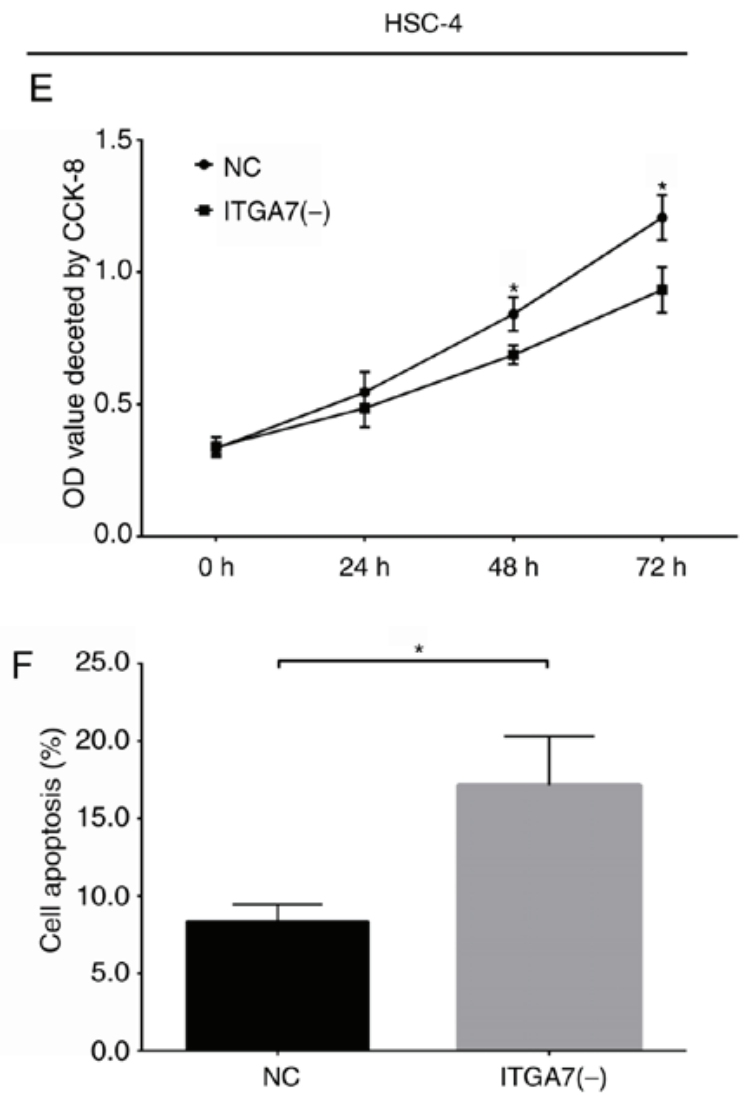

G
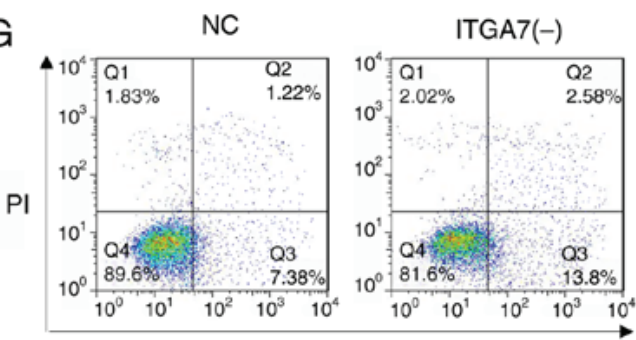

FITC-Annexin V

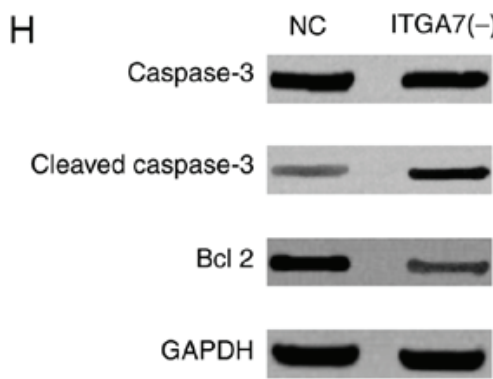

Figure 5. ITGA7 knockdown inhibits cell proliferation and promotes cell apoptosis in CAL-27 and HSC-4 cells. (A) Cell proliferation in ITGA7(-) and NC groups of CAL-27 cells was measured by CCK-8 assay. (B) Quantification and (C) representative plots from flow cytometry apoptosis analysis in CAL-27 cells. (D) Protein expression levels of apoptosis-related markers were detected by western blotting in CAL-27 cells. (E) Cell proliferation in ITGA7(-) and NC groups of HSC-4 cells was measured by CCK-8 assay. (F) Quantification and (G) representative plots from flow cytometry apoptosis analysis in HSC-4 cells. (H) Protein expression levels of apoptosis-related markers were detected by western blotting in HSC-4 cells. "P<0.05 and ${ }^{* *} \mathrm{P}<0.01$. ITGA7, integrin $\alpha 7$; NC, negative control; CCK-8, Cell Counting Kit-8; OD, optical density; PI, propidium iodide.

normal HOK cells (Fig. S3). Furthermore, ITGA7-positive cells and ITGA7-negative cells were sorted, and the protein expressions of ITGA7, CD24, CD44 and CD133 were detected in the separate cell populations by western blot analysis. The results demonstrated that ITGA7, CD44 and CD133 protein expressions were increased, while CD24 protein expression was decreased in ITGA7-positive cells compared with ITGA7-negative cells in both CAL-27 and HSC-4 cell lines (Fig. S4). These findings indicated that high ITGA7 expression was associated with high expression of CSC markers in TSCC cells. 
CAL-27
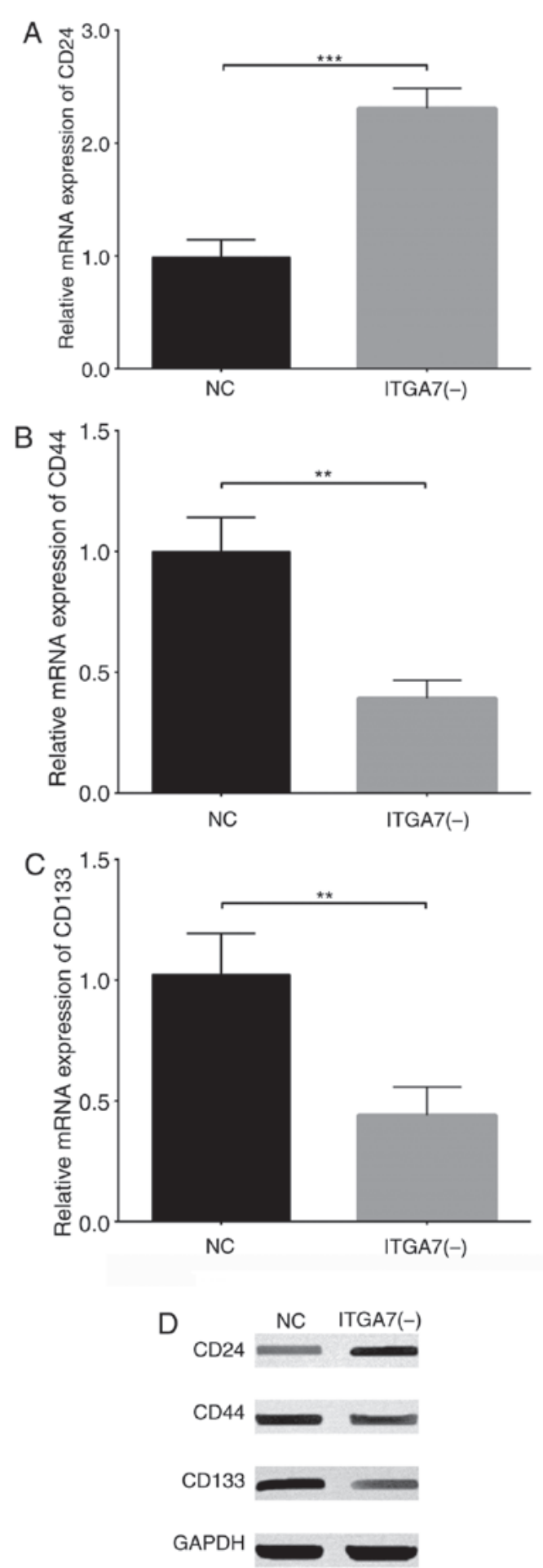
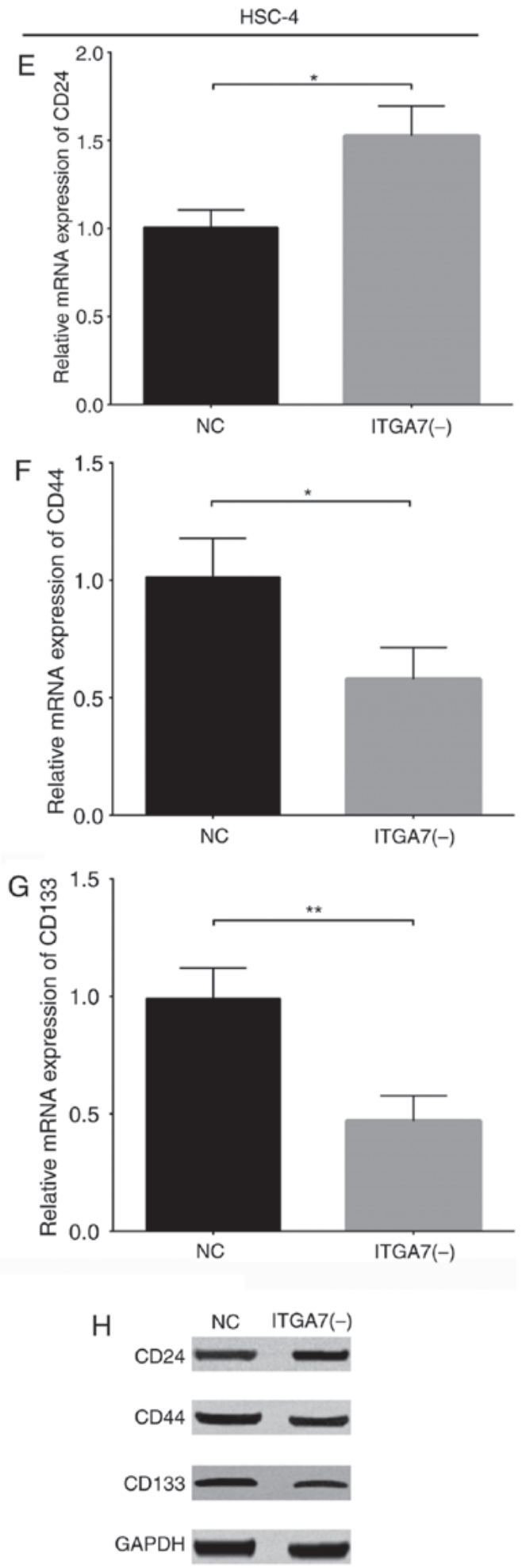

Figure 6. ITGA7 knockdown regulates common CSC markers in CAL-27 cells and HSC-4 cells. (A) CD24, (B) CD44 and (C) CD133 mRNA expression levels in ITGA7(-) and NC CAL-27 cells. (D) Protein expression levels of CSC markers in ITGA7(-) and NC CAL-27 cells. (E) CD24, (F) CD44 and (G) CD133 mRNA expression levels in ITGA7(-) and NC HSC-4 cells. (H) Protein expression levels of CSC markers in ITGA7(-) and NC HSC-4 cells. * $<<0.05,{ }^{* *} \mathrm{P}<0.01$ and ${ }^{* * * *} \mathrm{P}<0.001$. ITGA7, integrin $\alpha 7$; NC, negative control; CSC, cancer stemness cell.

Effects of ITGA7 knockdown on drug resistance to cisplatin in CAL-27 and HSC-4 cells. To further explore the role of ITGA7 in the stemness of TSCC cells, the effect of ITGA7 knockdown on drug resistance to cisplatin was investigated in CAL-27 and HSC- 4 cells. A CCK- 8 assay revealed that the relative cell viability decreased in the cisplatin + ITGA7(-) group compared with the cisplatin $+\mathrm{NC}$ group for CAL-27 cells $(\mathrm{P}<0.01$; Fig. 7A) and HSC-4 cells $(\mathrm{P}<0.05$; Fig. 7D). In addition, the rate of apoptosis increased in the cisplatin + ITGA7(-) group compared with the cisplatin + NC group of CAL-27 cells $(\mathrm{P}<0.01$; Fig. $7 \mathrm{~B}$ and $\mathrm{C})$ and HSC- 4 cells $(\mathrm{P}<0.05$; Fig. 7E and $\mathrm{F})$. These results suggested that ITGA7 
CAL-27
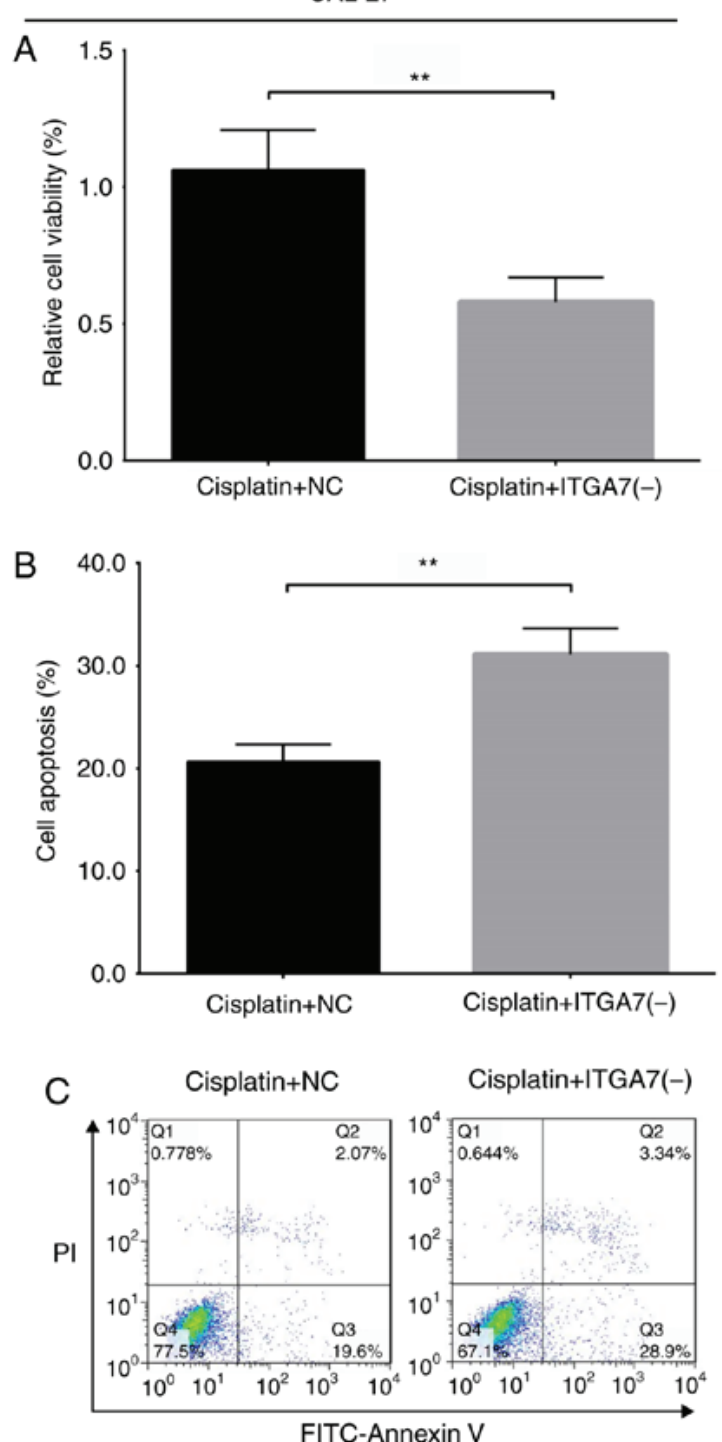

HSC-4
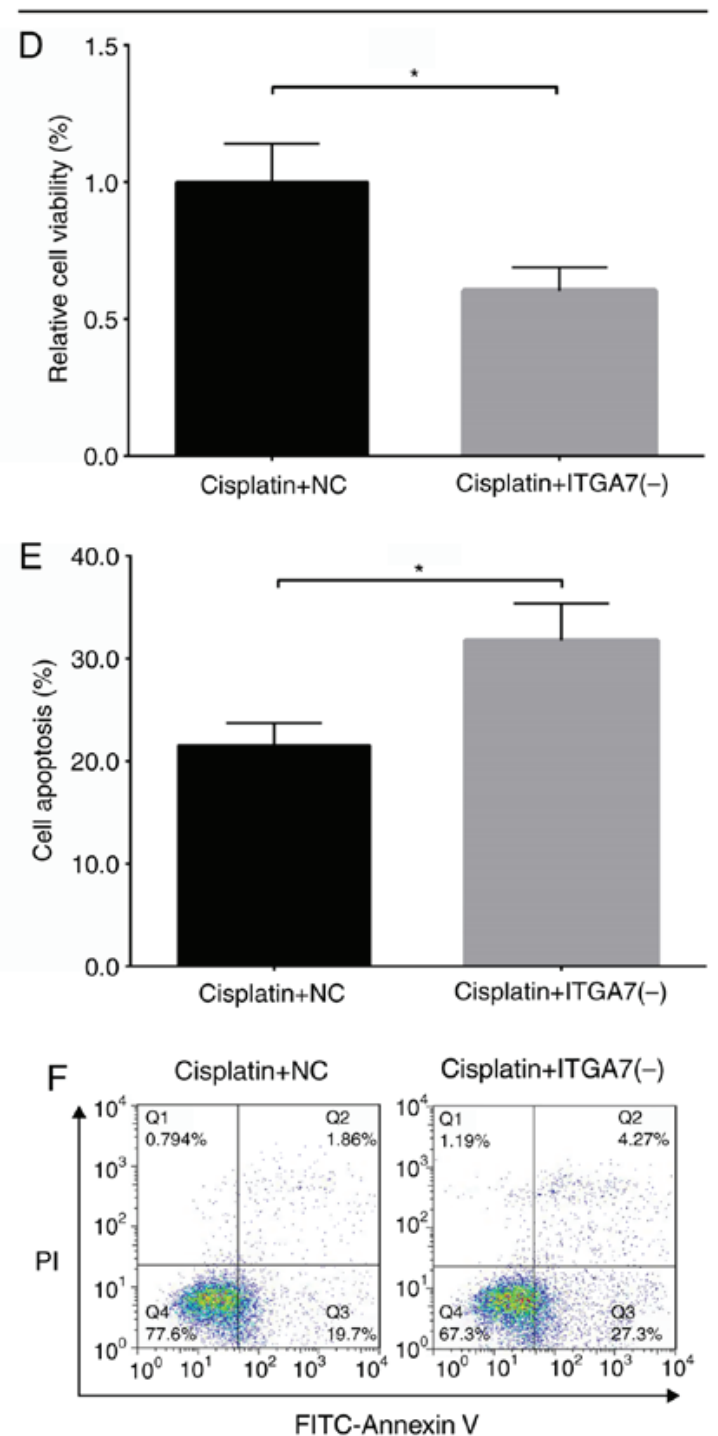

Figure 7. ITGA7 knockdown decreases drug resistance to cisplatin in CAL-27 cells and HSC-4 cells. (A) Relative cell viability and (B and C) apoptosis rate in cisplatin-treated ITGA7(-) and NC CAL-27 cells. (D) Relative cell viability and (E and F) apoptosis rate in cisplatin-treated ITGA7(-) and NC HSC-4 cells. ${ }^{*} \mathrm{P}<0.05$ and ${ }^{* *} \mathrm{P}<0.01$. ITGA7, integrin $\alpha 7$; NC, negative control; PI, propidium iodide.

knockdown decreased drug resistance to cisplatin in these cell lines.

Effects of ITGA7 knockdown on sphere formation ability of CAL-27 and HSC-4 cells. To further validate the effects of ITGA7 knockdown on the stemness of TSCC cells, sphere formation assays were performed in CAL-27 and HSC-4 cells. The results demonstrated that the sphere formation ability was reduced in the ITGA7(-) group compared with the NC group for CAL-27 cells ( $\mathrm{P}<0.01$; Fig. $8 \mathrm{~A}$ and $\mathrm{B}$ ) and HSC-4 cells $(\mathrm{P}<0.05$; Fig. $8 \mathrm{C}$ and $\mathrm{D})$. In addition, an extreme limited dilution assay revealed that the sphere formation ability was reduced in the ITGA7(-) group compared with the NC group for CAL-27 and HSC-4 cells (both $\mathrm{P}<0.001$; Table SI). These data indicated that ITGA7 knockdown decreased the sphere formation ability of CAL-27 and HSC-4 cells. Therefore, the aforementioned findings that ITGA7 regulated the expression of CSC markers, decreased drug resistance to cisplatin and suppressed sphere formation in CAL-27 and HSC-4 cells, indicated that ITGA7 knockdown may suppress TSCC cell stemness.

Potential of ITGA7 as a marker for CAL-27 and HSC-4 CSCs. In order to explore whether ITGA7 is a potential marker for TSCC stem cells, the expression of ITGA7 in drug-resistant (R-) CAL-27 and R-HSC-4 CSCs was detected, and sphere formation assays were performed. A CCK-8 assay demonstrated no notable differences between the R-CAL-27 + cisplatin (Fig. 9A) and R-HSC-4 + cisplatin groups (Fig. 9I) at 0, 24, 48 and $72 \mathrm{~h}$ $(\mathrm{P}>0.05)$, which indicated the successful generation of drug-resistant cells. In addition, a sphere formation assay was performed for CAL-27 (Fig. 9B) and HSC-4 (Fig. 9J) cells. The number of spheres/1,000 cells was increased in the R-CAL-27 ( $\mathrm{P}<0.01$; Fig. 9C) and R-HSC-4 ( $\mathrm{P}<0.01$; Fig. 9K) cells compared with the corresponding parental control cell lines. Next, spheres from the R-CAL-27 and R-HSC-4 groups were isolated by centrifugation, serving as CAL-27 and HSC-4 CSCs. To further validate the establishment of CAL-27 and HSC-4 CSCs, the 
CAL-27
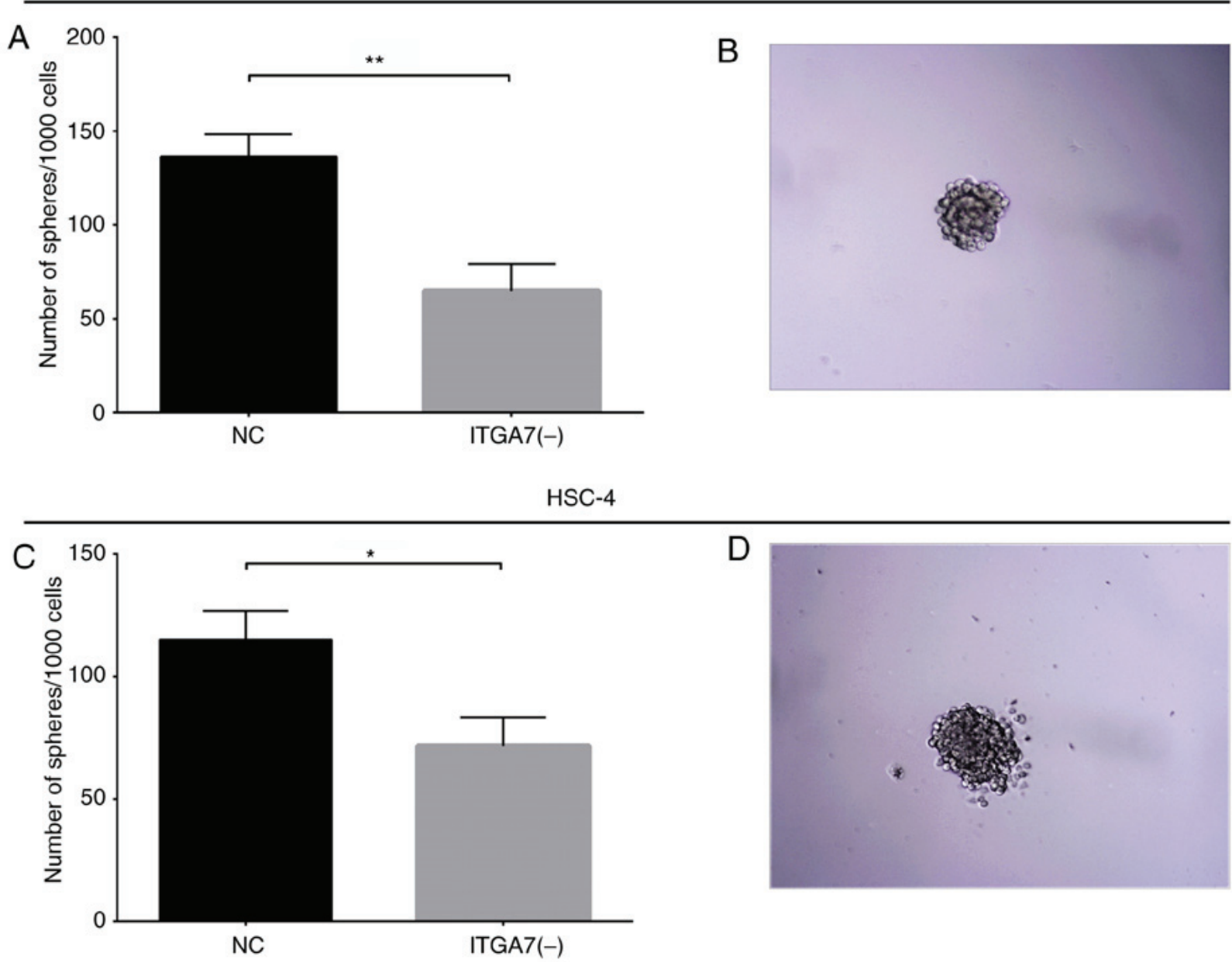

D

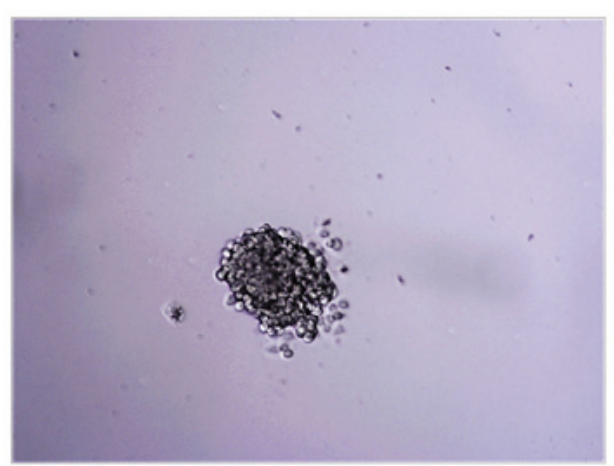

Figure 8. ITGA7 knockdown reduces the sphere formation efficiency of CAL-27 and HSC-4 cells. (A) Number of spheres/1,000 cells and (B) representative photomicrograph of a sphere (magnification, x200) in ITGA7(-) and NC CAL-27 cells. (C) Number of spheres/1,000 cells and (D) representative photomicrograph of a sphere (magnification, $\mathrm{x} 200$ ) in ITGA7(-) and NC HSC-4 cells. ${ }^{*} \mathrm{P}<0.05$ and ${ }^{* *} \mathrm{P}<0.01$. ITGA7, integrin $\alpha 7$; NC, negative control.

expression of common CSC markers was detected; the mRNA and protein expression levels of CD24 (Fig. 9D and H) were decreased, while those of CD44 ( $\mathrm{P}<0.01$; Fig. 9E and $\mathrm{H})$ and CD133 ( $\mathrm{P}<0.001$; Fig. 9F and $\mathrm{H}$ ) were increased in $\mathrm{CAL}-27$ CSCs compared with parental CAL-27 cells. Additionally, similar trends in the expression of CD24 ( $\mathrm{P}<0.01$; Fig. 9L and $\mathrm{P})$, CD44 $(\mathrm{P}<0.01$; and Fig. $9 \mathrm{M}$ and $\mathrm{P})$ and CD133 $(\mathrm{P}<0.001$; Fig. 9N and P) were observed for HSC-4 CSCs compared with parental HSC-4 cells, which suggested that CAL-27 and HSC-4 CSCs were successfully obtained. Following the successful establishment of CAL-27 and HSC-4 CSCs, the expression of ITGA7 was detected in the TSCC stem cells. The results revealed that the mRNA and protein expression levels of ITGA7 were increased in CAL-27 CSCs compared with parental CAL-27 cells $(\mathrm{P}<0.001$; Fig. $9 \mathrm{G}$ and $\mathrm{H})$, and in HSC-4 CSCs compared with parental HSC-4 cells $(\mathrm{P}<0.01$; Fig. $9 \mathrm{O}$ and $\mathrm{P})$. These findings indicated that ITGA7 may be a potential marker of TSCC stem cells.

\section{Discussion}

Integrins are a type of heterodimeric cell-surface adhesion molecule expressed in all nucleated cells (18). As one of the common integrins, ITGA7, which forms a heterodimer with integrin $\beta 1$, has been reported to be critical for tumor propagation and the regulation of CSC-associated properties (9-11). Controversial findings regarding the role of ITGA7 have been reported for patients with cancer, and this may be partly due to variations in the types of cancer and samples analyzed, or the inclusion and exclusion criteria applied for the enrollment of patients. However, the association between ITGA7 expression and the clinicopathological characteristics and prognosis of patients with TSCC was unknown $(9,11)$. In the present study, ITGA7 was determined to be upregulated in tumor tissues compared with in paired adjacent tissues, and its high expression was associated with increased pathological grade, higher $\mathrm{N}$ stage, and advanced TNM stage in patients with TSCC. The potential explanations for these observations are hypothesized as follows: i) ITGA7 promotes cell migration and invasion, and inhibits cell apoptosis by interacting with the epithelial-mesenchymal transition (EMT), focal adhesion kinase (FAK)/Akt or other signaling pathways to enhance tumor growth and metastasis, which may then be associated with increased pathological grade, higher $\mathrm{N}$ stage and advanced TNM stage in patients with TSCC $(11,19)$; and ii) ITGA7 could induce the stemness of TSCC cells to promote self-renewal 
CAL-27
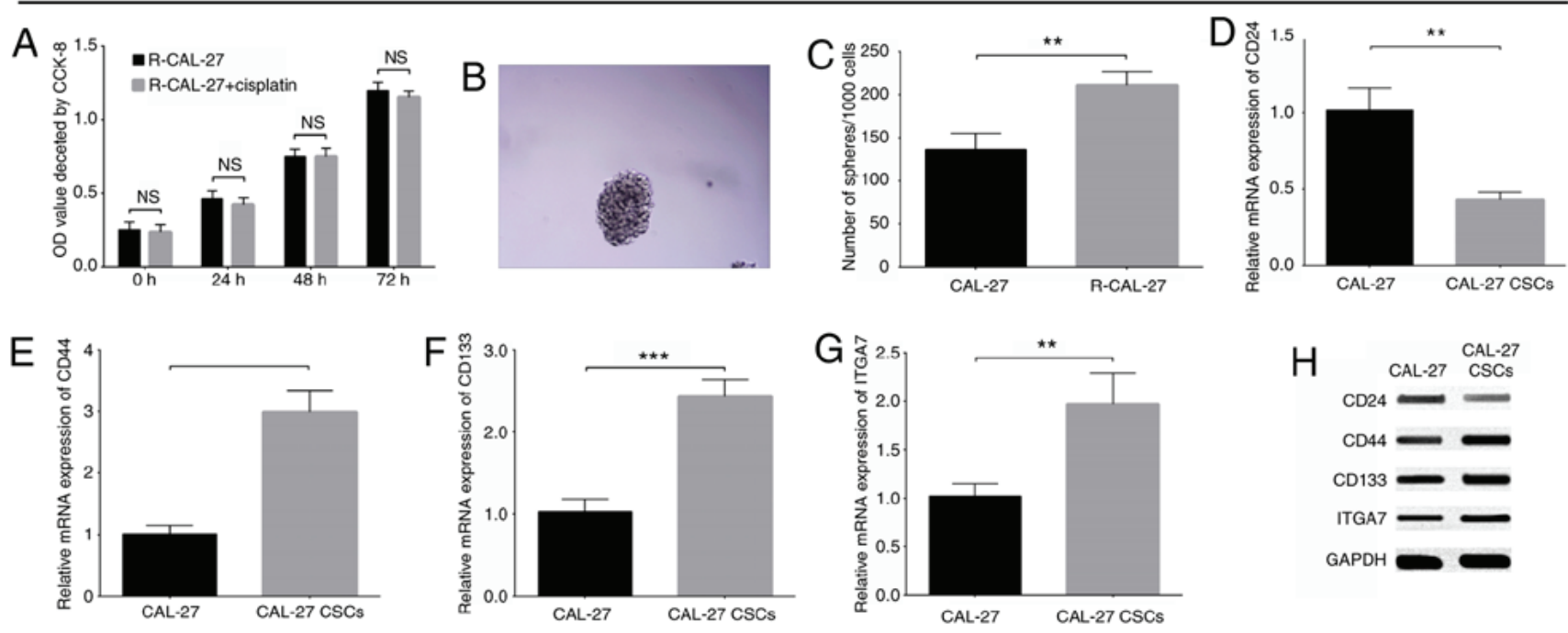

HSC-4
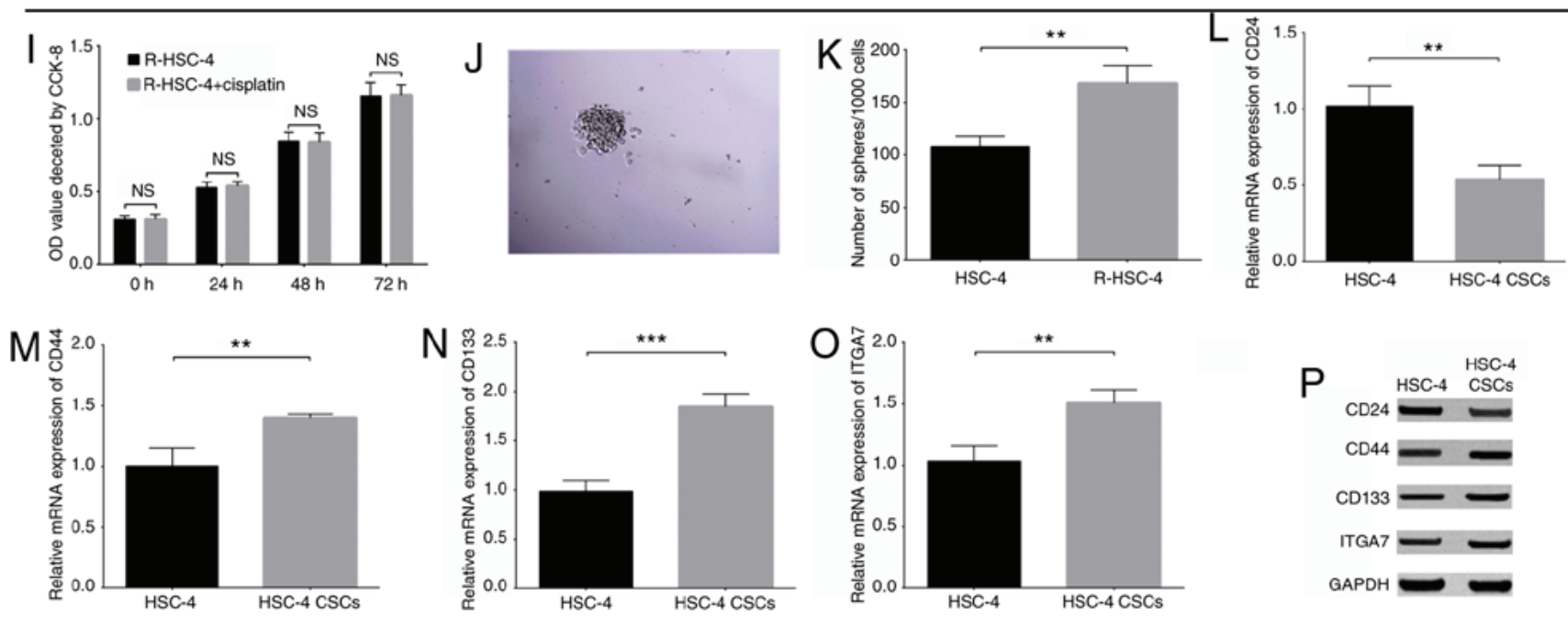

Figure 9. ITGA7 acts as a potential marker of CSCs in CAL-27 and HSC-4 cell lines. (A) CCK-8 assay of untreated and cisplatin-treated R-CAL-27 cells. (B) Representative photomicrograph of a sphere (magnification, x200) after lentivirus transfection in R-CAL-27 cells. (C) Number of spheres/1,000 cells in R-CAL-27 and parental CAL-27 cells. (D) CD24, (E) CD44, (F) CD133 and (G) ITGA7 mRNA expression levels in CAL-27 CSCs and CAL-27 parental cells. (H) Protein expression levels of CSC markers in CAL-27 CSCs and CAL-27 parental cells. (I) CCK-8 assay of untreated and cisplatin-treated R-HSC-4 cells. (J) Representative photomicrograph of a sphere (magnification, x200) after lentivirus transfection in R-HSC-4 cells. (K) Number of spheres/1,000 cells in R- HSC-4 and parental HSC-4 cells. (L) CD24, (M) CD44, (N) CD133 and (O) ITGA7 mRNA expression levels in HSC-4 CSCs and HSC-4 parental cells. (P) Protein expression levels of CSC markers in HSC-4 CSCs and HSC-4 parental cells. ${ }^{* * *} \mathrm{P}<0.01$ and ${ }^{* * * *} \mathrm{P}<0.001$. ITGA7, integrin $\alpha 7$; CSC, cancer stemness cell; CCK-8, Cell Counting Kit-8; R-, resistant; OD, optical density; NS, not significant.

and malignant phenotypes in cancer cells, which may then contribute to the poor prognosis of patients with TSCC. In addition, the potential of ITGA7 as an indicator of prognosis for patients with TSCC was investigated in the present study. The results demonstrated that upregulated ITGA7 expression was associated with poor OS in patients with TSCC, which was in accordance with previous studies that reported a negative correlation of ITGA7 expression with OS in patients with esophageal squamous cell carcinoma and glioma $(11,12)$. The possible reasons were as follows: i) ITGA7 was associated with worse clinicopathological characteristics, leading to poor prognosis and reduced OS in patients with TSCC; and ii) ITGA7 may promote drug resistance by inducing the stemness of TSCC cells. This could be associated with the unsatisfactory treatment outcomes and poor OS in patients with TSCC.
Recently, although limited data have been reported, investigations into the molecular mechanism underlying the effects of ITGA7 on the pathogenesis of carcinomas has been a novel research focus. For example, ITGA7 was determined to induce EMT promoting tumor metastasis, and to activate FAK/Akt signaling suppressing cell apoptosis, in esophageal squamous cell carcinoma (11). In lung cancer, ITGA7 induces cell migration and invasion by binding with S100 calcium binding protein P (19). The role of ITGA7 as a tumor promoter has been identified in several carcinomas; however, few studies have determined the effects of ITGA7 on the pathogenesis of TSCC. In the present study, RT-qPCR and western blot analyses were conducted to detect the mRNA and protein expression levels of ITGA7 in the human TSCC cell lines CAL-27, SCC-9, HSC-4, SCC-25 and the normal human oral keratinocytes cell line 
HOK. The present findings revealed that ITGA7 was upregulated in human TSCC cell lines compared with the normal HOK cell line. Subsequently, control shRNA and ITGA7-shRNA lentiviruses were constructed and transduced into CAL-27 and HSC-4 cells; CCK-8 and Annexin-V/PI staining assays were performed to investigate the effects of ITGA7 knockdown on the proliferation and apoptosis of TSCC cells. The results revealed that ITGA7 knockdown suppressed proliferation, but promoted apoptosis in CAL-27 and HSC-4 cells.

According to previous studies, ITGA7 has been reported as a functional CSC marker and is involved in the regulation of stem cell-like properties in several types of cancer. For instance, ITGA7 upregulates the expression of stemness-associated genes (including octamer-binding transcription factor 4, sex determining region Y-box 2, Nanog homeobox and CD90), and promotes the self-renewal ability of esophageal squamous cell carcinoma cells via the activation of the FAK-mediated signaling pathways (11). Furthermore, in vitro and in vivo experiments demonstrated that ITGA7 contributes to the growth and invasion of glioblastoma stem-like cells, potentially through interacting with laminin-induced outside-in signaling (12). These previous data indicated that ITGA7 may serve a critical role in regulating the stemness of cancer cells. In order to explore whether ITGA7 affected TSCC cell stemness, the present study investigated the effects of ITGA7 knockdown on the expression of common CSC markers (CD24, CD44 and

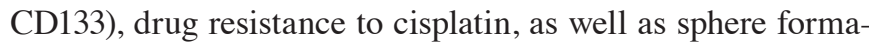
tion ability. The findings indicated that ITGA7 knockdown promoted the expression of $\mathrm{CD} 24$, while it downregulated that of CD44 and CD133, compared with the NC group of CAL-27 and HSC-4 cells. This suggested that ITGA7 knockdown regulated the expression of common CSC markers in TSCC cells. In addition, ITGA7 knockdown reduced drug resistance to cisplatin and decreased the sphere formation ability of CAL-27 and HSC-4 cells. These observations supported the findings of a recent study, which revealed that the apoptotic index of ITGA7-silenced esophageal squamous cell carcinoma cells increased after $48 \mathrm{~h}$ exposure to chemotherapeutic reagents (11). Furthermore, ITGA7-silenced KYSE180 and KYSE520 cells formed smaller and fewer spheroids compared with control cells (11). Therefore, the present results indicated that ITGA7 knockdown decreased TSCC cell stemness.

Based on the findings that ITGA7 regulated TSCC cell stemness, as evidenced by the regulation of CSC marker expression and the reductions in drug resistance and sphere formation, it was hypothesized that ITGA7 may be a novel potential marker of TSCC stem cells. Thus, CAL-27 and HSC-4 CSCs were generated by establishing drug-resistant cells, which were verfied via a sphere formation assay and analysis of CSC marker expression. The present study reported that the mRNA and protein expression levels of ITGA7 were increased in CAL-27 CSCs compared with parental CAL-27 cells; similar findings were obtained for HSC-4 CSCs. These results indicated that ITGA7 may act as a potential marker for TSCC stem cells. Therefore, the findings of the present study provided novel and comprehensive insight into the molecular mechanisms underlying the role of ITGA7 in the pathogenesis of TSCC.

In summary, ITGA7 was determined to be upregulated in tumor tissues, and its high expression was associated with worse clinicopathological characteristics and poor overall survival in patients with TSCC. In addition, ITGA7 knockdown suppressed proliferation and stemness, but promoted apoptosis, in TSCC cells in vitro. The present findings indicated that ITGA7 may serve as a potential marker for TSCC stem cells.

\section{Acknowledgements}

Not applicable.

\section{Funding}

This study was supported by the National Natural Science Foundation of China (grant no. 81672827).

\section{Availability of data and materials}

All data generated or analyzed during this study are included in this published article.

\section{Authors' contributions}

CY contributed to study conception and design, and reviewed the manuscript. ZL and CY provided the study materials and revised the manuscript. ZL and YY were responsible for data analysis and manuscript writing. All authors approved the final manuscript.

\section{Ethics approval and consent to participate}

The present study was approved by the Institutional Review Board of The Second Affiliated Hospital of Harbin Medical University, and written informed consent was obtained from all patients.

\section{Patient consent for publication}

Not applicable.

\section{Competing interests}

The authors declare that they have no competing interests.

\section{References}

1. Almangush A, Heikkinen I, Mäkitie AA, Coletta RD, Läärä E, Leivo I and Salo T: Prognostic biomarkers for oral tongue squamous cell carcinoma: A systematic review and meta-analysis. Br J Cancer 117: 856-866, 2017.

2. Siegel RL, Miller KD and Jemal A: Cancer statistics, 2018. CA Cancer J Clin 68: 7-30, 2018.

3. Pfister DG, Ang KK, Brizel DM, Burtness BA, Busse PM, Caudell JJ, Cmelak AJ, Colevas AD, Dunphy F, Eisele DW, et al: Head and neck cancers, version 2.2013. Featured updates to the NCCN guidelines. J Natl Compr Canc Netw 11: 917-923, 2013.

4. Gore SM, Crombie AK, Batstone MD and Clark JR: Concurrent chemoradiotherapy compared with surgery and adjuvant radiotherapy for oral cavity squamous cell carcinoma. Head Neck 37: 518-523, 2015.

5. Iyer NG, Tan DS, Tan VK, Wang W, Hwang J, Tan NC, Sivanandan R, Tan HK, Lim WT, Ang MK, et al: Randomized trial comparing surgery and adjuvant radiotherapy versus concurrent chemoradiotherapy in patients with advanced, nonmetastatic squamous cell carcinoma of the head and neck: 10-year update and subset analysis. Cancer 121: 1599-1607, 2015. 
6. Goel HL, Li J, Kogan S and Languino LR: Integrins in prostate cancer progression. Endocr Relat Cancer 15: 657-664, 2008.

7. Juan-Rivera MC and Martinez-Ferrer M: Integrin inhibitors in prostate cancer. Cancers (Basel) 10: E44, 2018.

8. Tan LZ, Song Y, Nelson J, Yu YP and Luo JH: Integrin alpha7 binds tissue inhibitor of metalloproteinase 3 to suppress growth of prostate cancer cells. Am J Pathol 183: 831-840, 2013.

9. Bhandari A, Xia E, Zhou Y, Guan Y, Xiang J, Kong L, Wang Y, Yang $\mathrm{F}$, Wang $\mathrm{O}$ and Zhang $\mathrm{X}$ : ITGA7 functions as a tumor suppressor and regulates migration and invasion in breast cancer. Cancer Manag Res 10: 969-976, 2018.

10. Ren B, Yu YP, Tseng GC, Wu C, Chen K, Rao UN, Nelson J, Michalopoulos GK and Luo JH: Analysis of integrin alpha7 mutations in prostate cancer, liver cancer, glioblastoma multiforme, and leiomyosarcoma. J Natl Cancer Inst 99: 868-880, 2007.

11. Ming XY, Fu L, Zhang LY, Qin YR, Cao TT, Chan KW, Ma S, Xie D and Guan XY: Integrin $\alpha 7$ is a functional cancer stem cell surface marker in oesophageal squamous cell carcinoma. Nat Commun 7: 13568, 2016.

12. Haas TL, Sciuto MR, Brunetto L, Valvo C, Signore M, Fiori ME, di Martino S, Giannetti S, Morgante L, Boe A, et al: Integrin $\alpha 7$ is a functional marker and potential therapeutic target in glioblastoma. Cell Stem Cell 21: 35-50.e39, 2017.

13. Carrasco-Garcia E, Auzmendi-Iriarte J and Matheu A: Integrin $\alpha 7$ : A novel promising target in glioblastoma stem cells. Stem Cell Investig 5: 2, 2018.
14. Abd El hafez A and El-Hadaad HA: Immunohistochemical expression and prognostic relevance of Bmi-1, a stem cell factor, in epithelial ovarian cancer. Ann Diagn Pathol 18: 58-62, 2014.

15. Hu Y and Smyth GK: ELDA: Extreme limiting dilution analysis for comparing depleted and enriched populations in stem cell and other assays. J Immunol Methods 347: 70-78, 2009.

16. Dontu G, Abdallah WM, Foley JM, Jackson KW, Clarke MF, Kawamura MJ and Wicha MS: In vitro propagation and transcriptional profiling of human mammary stem/progenitor cells. Genes Dev 17: 1253-1270, 2003.

17. Livak KJ and Schmittgen TD: Analysis of relative gene expression data using real-time quantitative PCR and the 2(-Delta Delta C(T)) method. Methods 25: 402-408, 2001.

18. Hynes RO: Integrins: Bidirectional, allosteric signaling machines. Cell 110: 673-687, 2002.

19. Hsu YL, Hung JY, Liang YY, Lin YS, Tsai MJ, Chou SH, Lu CY and Kuo PL: S100P interacts with integrin $\alpha 7$ and increases cancer cell migration and invasion in lung cancer. Oncotarget 6: 29585-29598, 2015.

(i) (9) This work is licensed under a Creative Commons EY NO NO Attribution-NonCommercial-NoDerivatives 4.0 International (CC BY-NC-ND 4.0) License. 